\title{
Investigation of a drag reduction on a circular cylinder in rotary oscillation
}

\author{
By D. SHIELS AND A. LEONARD \\ Graduate Aeronautics Laboratories, California Institute of Technology, \\ Pasadena CA 91125, USA
}

(Received 28 October 1998 and in revised form 13 July 2000)

Drag reduction in two-dimensional flow over a circular cylinder, achieved using rotary oscillation, was investigated with computational simulations. In the experiments of Tokumaru \& Dimotakis (1991), this mechanism was observed to yield up to $80 \%$ drag reduction at $R e=15000$ for certain ranges of frequency and amplitude of sinusoidal rotary oscillation. Simulations with a high-resolution viscous vortex method were carried out over a range of Reynolds numbers (150-15000) to explore the effects of oscillatory rotational forcing. Significant drag reduction was observed for a rotational forcing which had been very effective in the experiments. The impact of the forcing is strongly Reynolds number dependent. The cylinder oscillation appears to trigger a distinctive shedding pattern which is related to the Reynolds number dependence of the drag reduction. It appears that the source of this unusual shedding pattern and associated drag reduction is vortex dynamics in the boundary layer initiated by the oscillatory cylinder rotation. The practical efficiency of the drag reduction procedure is also discussed.

\section{Introduction}

Study of the flow over an oscillating circular cylinder has primarily focused on linear oscillations (i.e. inline or transverse), as for example evidenced in the review paper of Griffin \& Hall (1991). Among the few studies into flow over a cylinder undergoing rotary oscillation, Tokumaru \& Dimotakis (1991) found significant drag reduction could be achieved with such a control mechanism. For Reynolds number $R e=15000$ flow, the cylinder was subjected to a sinusoidal rotation (cf. figure 1)

$$
\Omega^{*}\left(t^{*}\right)=\frac{\dot{\theta} R}{U_{\infty}}=\Omega_{1}^{*} \sin \left(\pi S_{f} t^{*}\right)
$$

where

$$
t^{*}=\frac{t U_{\infty}}{R}
$$

and the forcing Strouhal number is given by

$$
S_{f}=\frac{2 f R}{U_{\infty}} .
$$

For $\Omega_{1}^{*}=2$, a drag reduction was measured from an unforced drag coefficient $c_{D} \approx 1.4$ to 0.2 when $S_{f}=1$, where

$$
c_{D}=\frac{\text { Drag }}{\frac{1}{2} \rho U_{\infty}^{2} D} .
$$




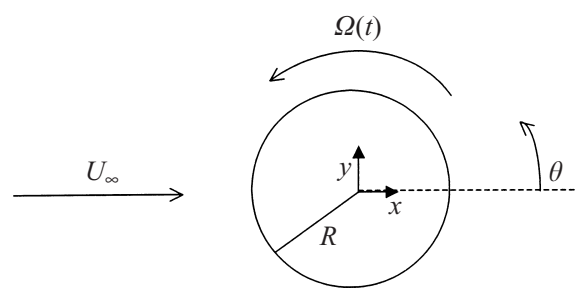

FIGURE 1. The flow under consideration.

Flow visualization indicated that the vortex shedding 'locked-on' to the forcing frequency, leading to smaller shed vortices and a narrowing of the width of the wake. It was also noted that a similar though less pronounced behaviour was evident at $\operatorname{Re}=3300$.

The results raise interesting questions concerning the vortex dynamics underlying the wake development. For example, the vortices are shed at a time in the rotational cycle that is counter-intuitive. A vortex structure appears and separates from the cylinder on the side where the body motion follows the free-stream direction, whereas one might expect separation on the side where the body motion opposes the free stream. In general the detailed mechanism behind this shedding process and the subsequent drag reduction is unknown. Other studies using rotational forcing, including Okajima, Takata \& Asanuma (1975), Wu, Mo \& Vakili (1989), Ou (1991), Filler, Marston \& Mih (1991), El-Refaee (1995), Zakharenkov (1996), Chou (1997), and Baek \& Sung (1998), consider different issues at lower Reynolds numbers and for less vigorous rotations. Taneda (1978) presents curious flow visualizations suggesting that complete suppression of separation for much lower Reynolds numbers can be achieved with a large-amplitude rotational forcing, although there has been no subsequent validation of these results.

The motivation behind the present computational study is to investigate the phenomenon underlying the observations in Tokumaru \& Dimotakis (1991). The accuracy of the drag measurements obtained from wake surveys in the experiments can also be assessed. Although the simulations presented below are for two-dimensional flow, and flow past a fixed cylinder at $R e=15000$ is three-dimensional, the forcing could impose strong two-dimensionality on the near wake. In any case, the two-dimensional simulations should reveal the critical physics in the boundary layers and in the near wake of the more complex three-dimensional flow.

\section{Computational method}

Simulations were performed with a viscous vortex method capable of achieving high resolution. A continuous vorticity field can be represented by a collection of Gaussian distributions of vorticity referred to as vortex particles,

$$
\omega(\boldsymbol{x}, t)=\sum_{i=1}^{N} \frac{1}{2 \pi \sigma_{i}(t)^{2}} \Gamma_{i}(t) \exp \left[-\frac{1}{2}\left(\frac{\left|\boldsymbol{x}-\boldsymbol{x}_{i}(t)\right|}{\sigma_{i}(t)}\right)^{2}\right] .
$$

The two-dimensional vortex method tracks the evolution of a vorticity field, governed by

$$
\frac{\mathrm{D} \omega}{\mathrm{D} t}=v \nabla^{2} \omega,
$$


by creating vortex particles at the viscous cylinder boundary in order to satisfy the velocity boundary conditions and convecting those elements according to

$$
\boldsymbol{u}(\boldsymbol{x}, t)=-\frac{1}{2 \pi} \iint \frac{\left(\boldsymbol{x}-\boldsymbol{x}^{\prime}\right) \times \omega \hat{z}}{\left|\boldsymbol{x}-\boldsymbol{x}^{\prime}\right|^{2}} \mathrm{~d} \boldsymbol{x}^{\prime}+\nabla \Phi .
$$

The potential function $\Phi$ includes the irrotational free stream.

Two different approaches were used in this study to include viscous effects. The first, used to achieve the highest resolution, is the particle strength exchange technique (cf. Degond \& Mas Gallic (1989)), in which the Laplacian is approximated by an integral operator

$$
\nabla^{2} \omega(\boldsymbol{x}) \approx \frac{4}{\pi \varepsilon^{4}} \iint\left(\omega\left(\boldsymbol{x}^{\prime}\right)-\omega(\boldsymbol{x})\right) \exp \left(-\frac{\left|\boldsymbol{x}-\boldsymbol{x}^{\prime}\right|^{2}}{\varepsilon^{2}}\right) \mathrm{d} \boldsymbol{x}^{\prime},
$$

where $\varepsilon^{2}=O(v \Delta t)$. This relationship is used to update the particle strengths $\Gamma_{i}$ to accurately simulate diffusion. The particle core sizes are kept uniform both spatially and temporally:

$$
\sigma_{i}(t)=\text { constant }
$$

The second approach is based on the core expansion technique proposed by Leonard (1980), where diffusion is simulated by allowing the core sizes to evolve according to an exact solution to the heat equation

$$
\frac{\mathrm{d} \sigma^{2}}{\mathrm{~d} t}=2 v
$$

Particle splitting and merging, similar to that proposed by Rossi $(1996,1997)$, is used to alleviate the convergence concerns raised by Greengard (1985) and yield reasonable computational efficiency. The core expansion method provides better efficiency than the particle strength exchange technique (with constant core sizes) while maintaining a high level of accuracy.

The no-slip condition on tangential velocity at the boundary,

$$
(\boldsymbol{u} \cdot \hat{\boldsymbol{\theta}})_{\text {boundary }}=\Omega(t) R,
$$

is satisfied by introducing a vortex sheet on the cylinder surface to supplement the field of vortex particles. This vortex sheet is diffused into the flow field as described in Koumoutsakos, Leonard \& Pepin (1994) and thus transferred to the particle field. By satisfying the no-slip condition, the no-through flow condition

$$
(\boldsymbol{u} \cdot \hat{\boldsymbol{r}})_{\text {boundary }}=0
$$

is automatically met (cf. Spalart 1982).

The particle strength exchange vortex method has been well-validated for flow over a fixed circular cylinder by Koumoutsakos \& Leonard (1995), for a rotationally oscillating cylinder by Shiels, Leonard \& Stagg (1995), and for flow normal to a flat plate by Koumoutsakos \& Shiels (1996). A more thorough exposition of the vortex methods utilized in this study is provided in Shiels (1998).

\section{Results and discussion}

Accurate computation at $R e=15000$ is extremely challenging owing to the fine scales of relevance. Thus, two sets of simulations were run in this study. The first used the particle strength exchange technique, which provided a very high level of 

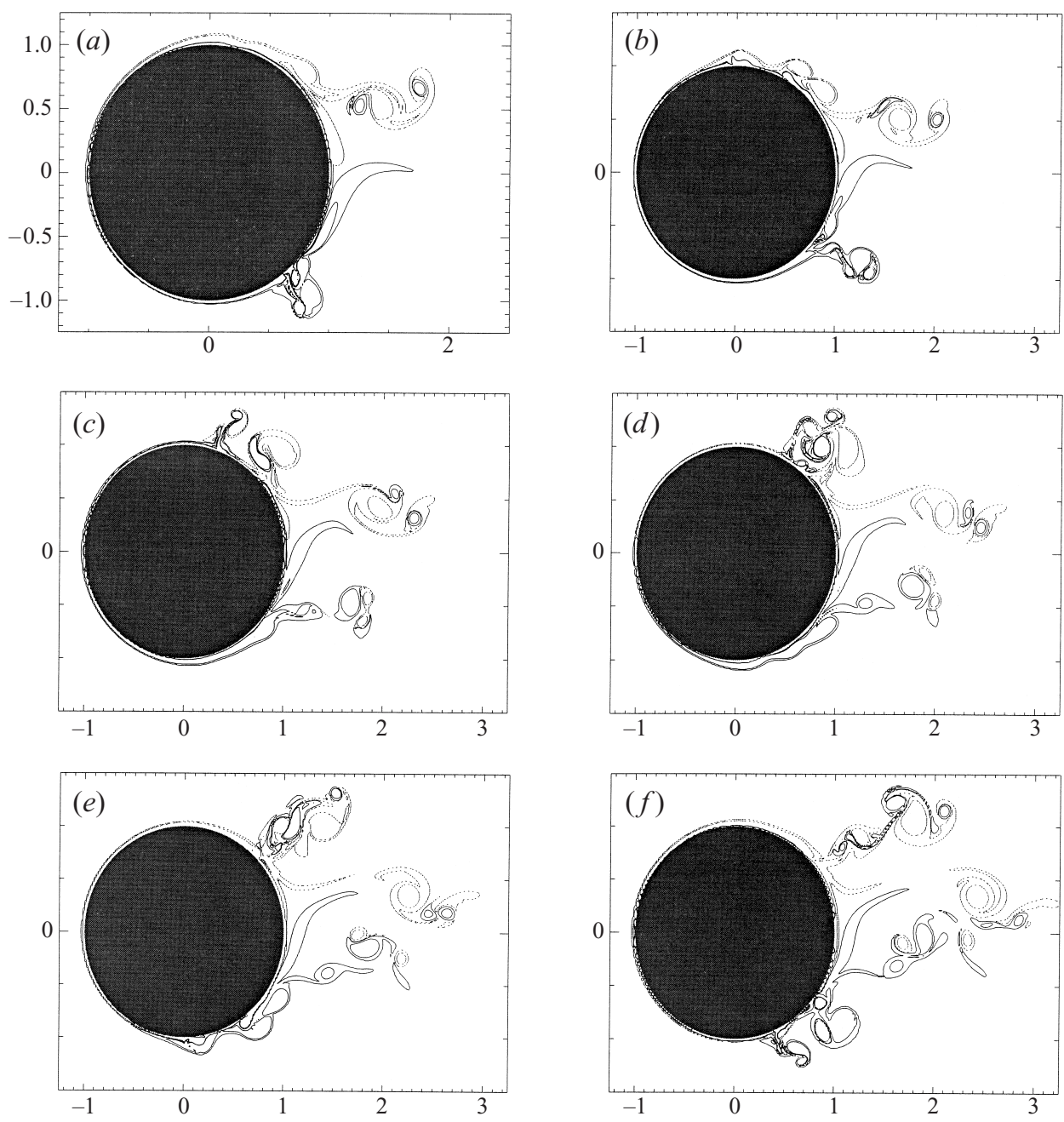

FIGURE 2. The vorticity field for $R e=15000$ flow, $\Omega^{*}=2 \sin \left(\pi t^{*}\right)$, contours at $\omega^{*}= \pm 1, \pm 10$, $\Delta x^{*} \approx 0.0015, \Delta t^{*}=0.004$. Positive vorticity is shown with solid lines, negative with dashed lines. (a) $t^{*}=3.15,(b)$ 3.6, (c) 4.0, (d) 4.3, (e) 4.6, (f) 5.0.

resolution but involved excessive computation after the initial stages of the flow. The second used the core expansion method, which permitted variable spatial resolution and thus facilitated simulation to later stages of the flows. The results obtained with the core expansion technique are validated against the particle strength exchange simulations to verify that sufficient resolution is obtained.

\subsection{High-resolution, short-time simulations of $R e=15000$ flow, $\Omega_{1}^{*}=2, S_{f}=1$}

The first set of simulations utilized the particle strength exchange (PSE) method discussed above. Owing to the fine scales in the boundary layer and the need to maintain uniform resolution with the PSE approach, it was not feasible to extend simulations beyond 2.5 diameters of downstream motion from an impulsive start, despite the use of a 256 processor Cray T3D. Still, the dominant features of the flow appeared to establish themselves rapidly and thus were apparent in these simulations. The only scenario tested with the PSE vortex method was $R e=15000$ flow with 

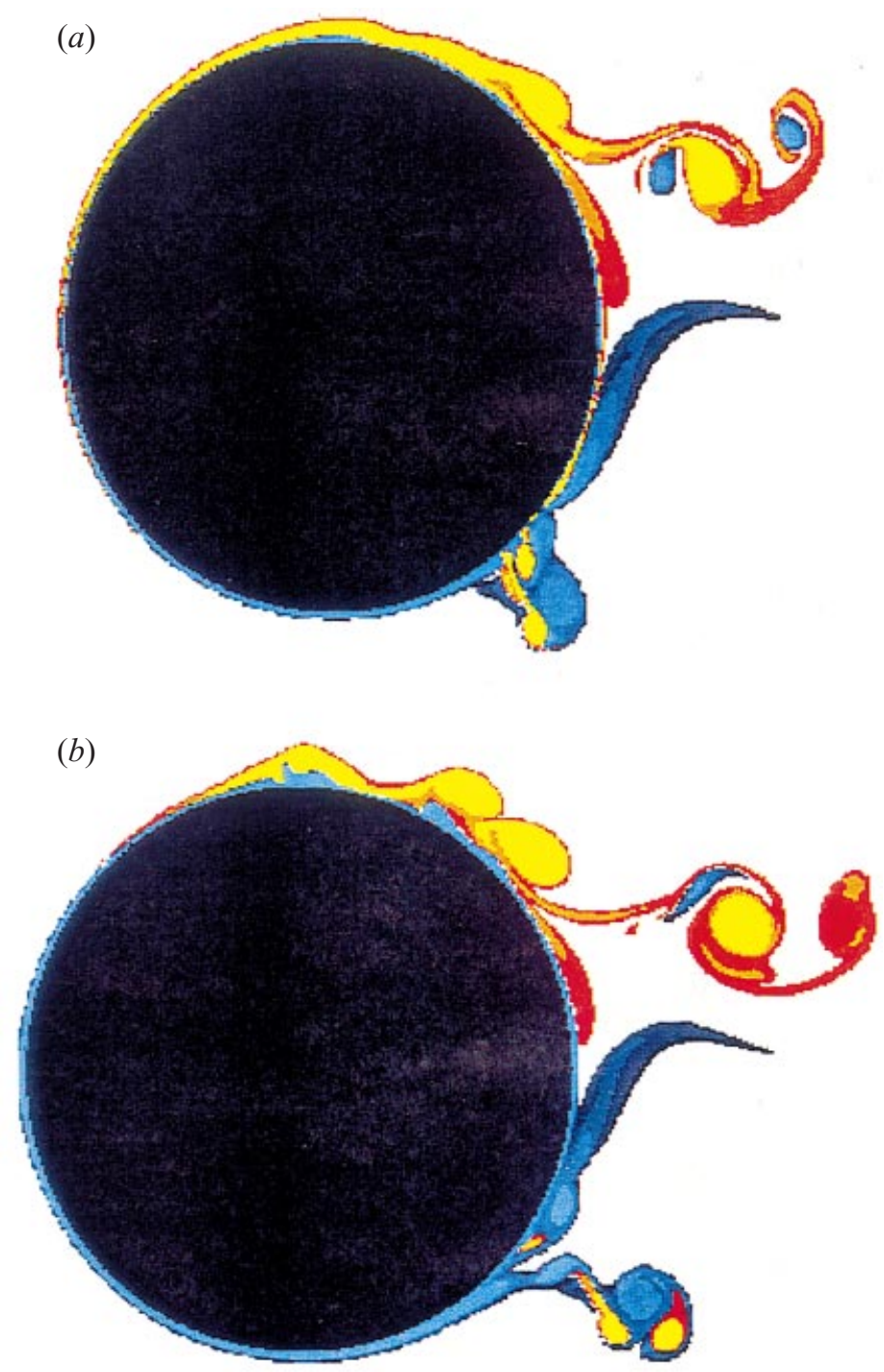

FIGURE $3(a, b)$. For caption see page 303 .

the cylinder oscillation given by $\Omega^{*}=2 \sin \left(\pi t^{*}\right)$. For this case, Tokumaru (1991) measured a drag coefficient of $c_{D} \approx 0.2$ (whereas $c_{D} \approx 1.4$ was found in the unforced case). A 'narrowing' of the transverse extent of the wake was also observed in the experiments, suggesting that an effective delay in mean separation (i.e. separation at smaller $|\theta|)$ coincided with the drag reduction.

A simulation was taken to $t^{*}=5$ (2.5 diameters of downstream motion), which required 40 hours of use of all 256 processors and resulted in a field with 1.7 million computational particles during the final stage of the simulations. The flow was resolved to $\Delta x^{*} \approx 0.0015$ (where $x^{*}=x / R$ ) and $\Delta t^{*}=0.004$. A higher-resolution simulation presented below indicates that although some relevant scales exist at finer resolution, the dominant behaviour seems to be captured in these less-intensive computations.

Vorticity fields presented in figures 2 and 3 reveal flow phenomena that could explain the findings in Tokumaru's (1991) experiment. It appears that during each 

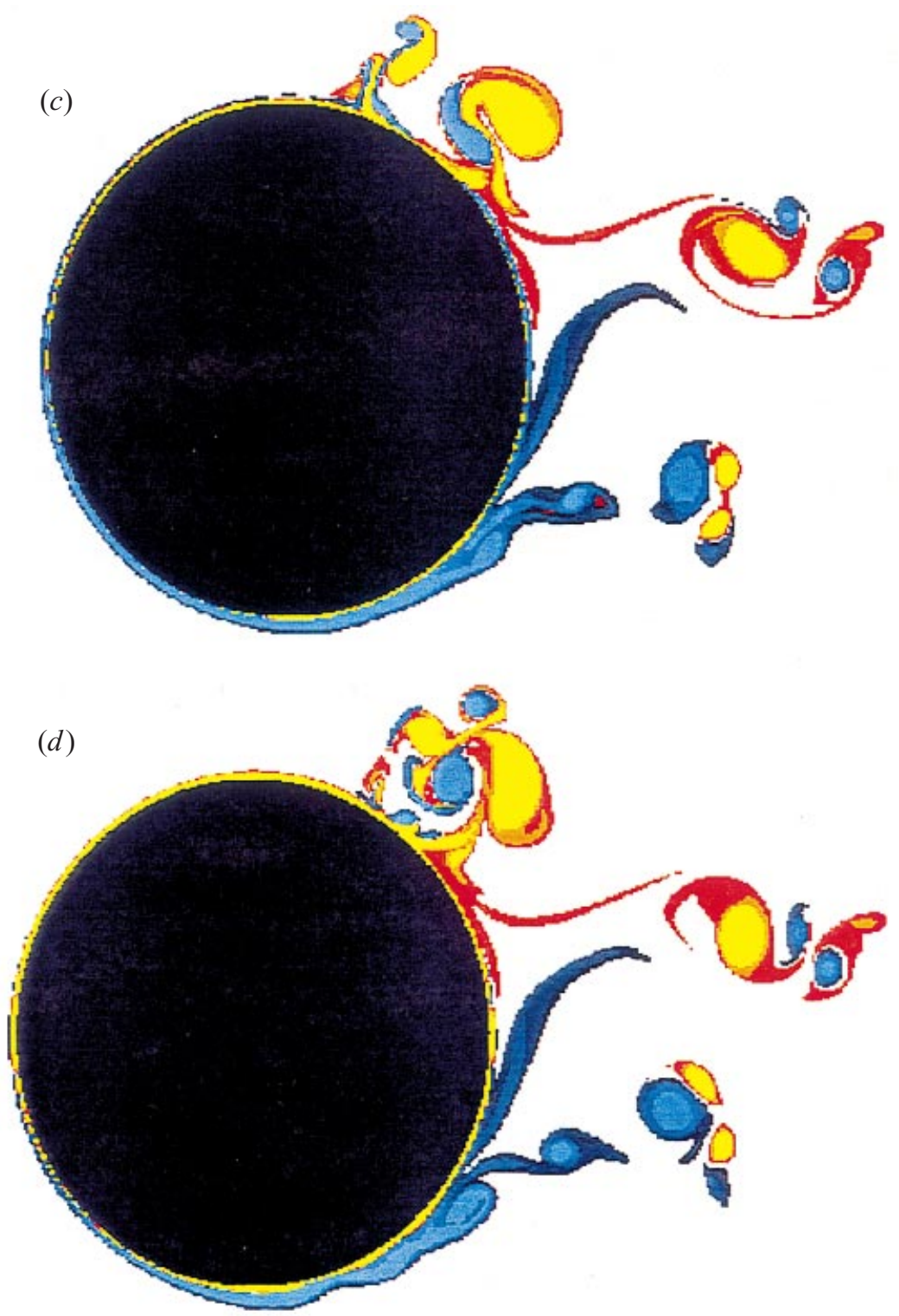

FIGURE $3(c, d)$. For caption see opposite.

half forcing cycle (every $\Delta t^{*}=1$ ) a multipole vorticity structure is generated on alternating sides of the cylinder that is rapidly ejected from the boundary layer and quickly convects downstream. In figure 2(a), two structures in opposite halves of the flow are characterized by significant levels of both signs of vorticity. The structure in the upper half has already entered the wake while the lower structure lies just off the body surface. At this time, the body is rotating clockwise in accordance with its prescribed motion. Thus the boundary at $y^{*}=1$ is moving in the same direction as the free stream, which classically suggests that boundary layer separation would be delayed in this region. Conversely, boundary layer separation would be expected to be encouraged on the lower half $\left(y^{*}=1\right)$ because the body is moving in opposition to the free stream. 

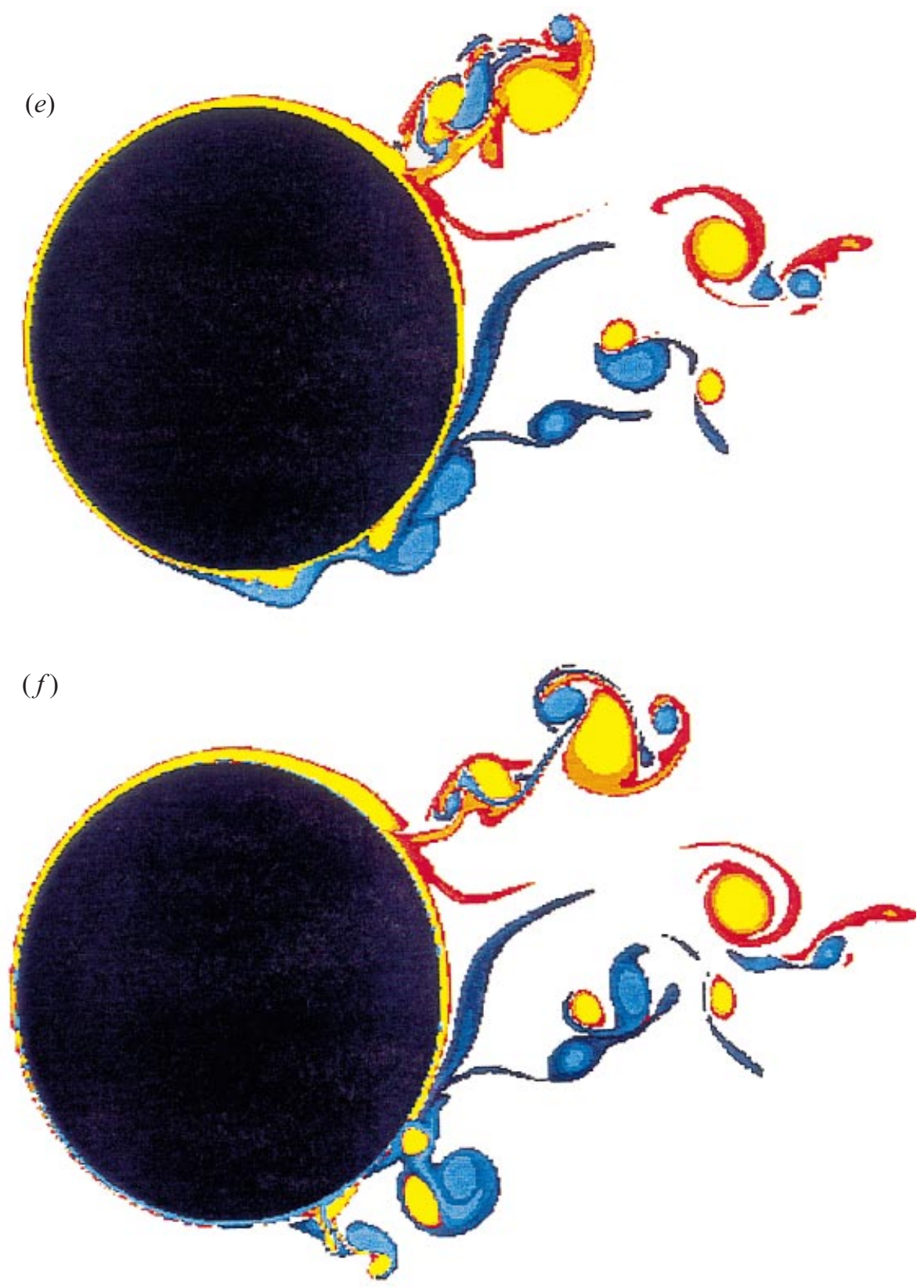

Figure 3. Colour vorticity plots at $(a) t^{*}=3.15,(b)$ 3.6, (c) 4.0, (d) 4.3, (e) 4.6, (f) 5.0.

Thus, the behaviour apparent in figure $2(b)$ is somewhat surprising. While the body has rotated clockwise, a good deal of vorticity is seen lifting off the body on the upper-half, where suppression of separation was expected. Meanwhile, the boundary layer region appears quiescent on the lower half where separation enhancement might be expected. Note also that the original multipole vortex structure on the upper half continues to move downstream and the multipole structure on the lower half has risen sharply off the body and downstream. The boundary layer dynamics in these 


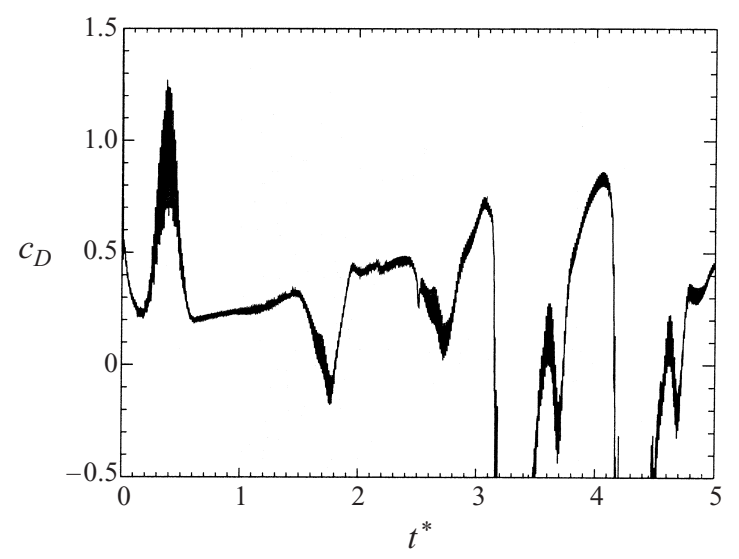

FIgURE 4. Drag for $R e=15000$ simulation, $\Omega^{*}=2 \sin \left(\pi t^{*}\right), \Delta x^{*} \approx 0.0015, \Delta t^{*}=0.004$.

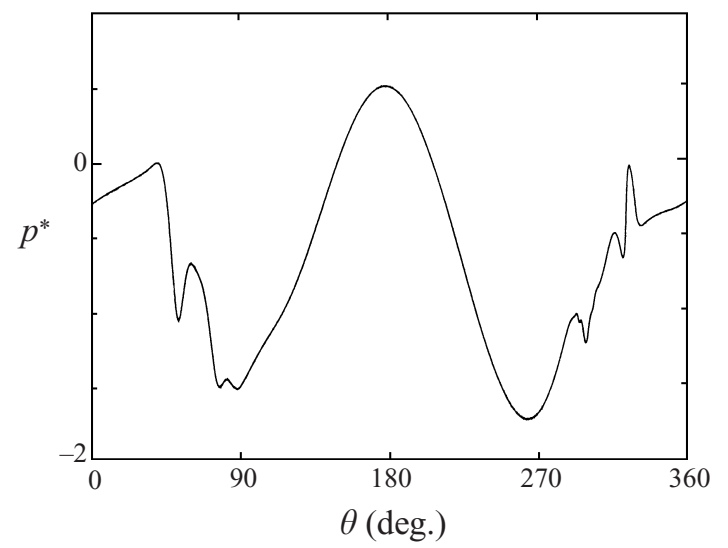

Figure 5. Pressure distribution on the cylinder surface for $R e=15000$ flow, $\Omega^{*}=2 \sin \left(\pi t^{*}\right)$, at $t^{*}=5$. The reference value $p^{*}\left(\theta=180^{\circ}\right)=0.5$ is chosen, which would be the upstream stagnation point in the potential flow over a fixed cylinder.

multipole formation regions are examined in some detail below. It is apparent that the oscillation produces opposing layers of vorticity above the wall. The layers have increasing strength in the steamwise direction, leading to a bursting event, which can trigger multipole generation.

When the body has completed its clockwise rotation, in figure 2(c), the original structures have clearly shed from the immediate vicinity of the body and entered the wake and two new multipole vortex structures have risen during this half-cycle from the boundary layer on the upper half of the body. In figures $2(d), 2(e)$, and $2(f)$, the same cycle repeats itself, but on the opposite half of the cylinder, with counter-clockwise rotation of the body. In these plots, note the new vortex structures formed during the cycle of motion and their subsequent detachment from the body and convection into the near wake. They are considerably more complex than the original two structures apparent in figure $2(a)$.

The simulation was stopped at $t^{*}=5$ as the computational expense, which increases with the wake growth, was becoming excessive. However, trends can clearly be seen developing by this time. The drag coefficient, given in figure 4 , is at lower values than the experimental measurements for a non-rotating cylinder of $c_{D} \approx 1.4$. The 


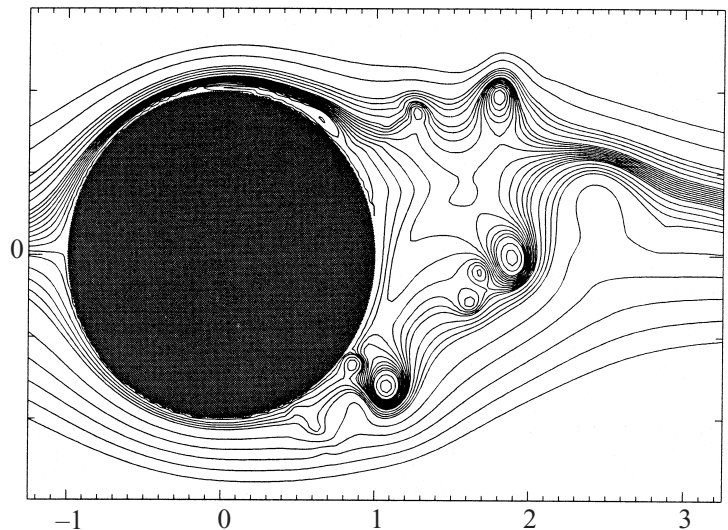

Figure 6. Streamlines for $R e=15000$ flow, $\Omega^{*}=2 \sin \left(\pi t^{*}\right)$, at $t^{*}=5$.

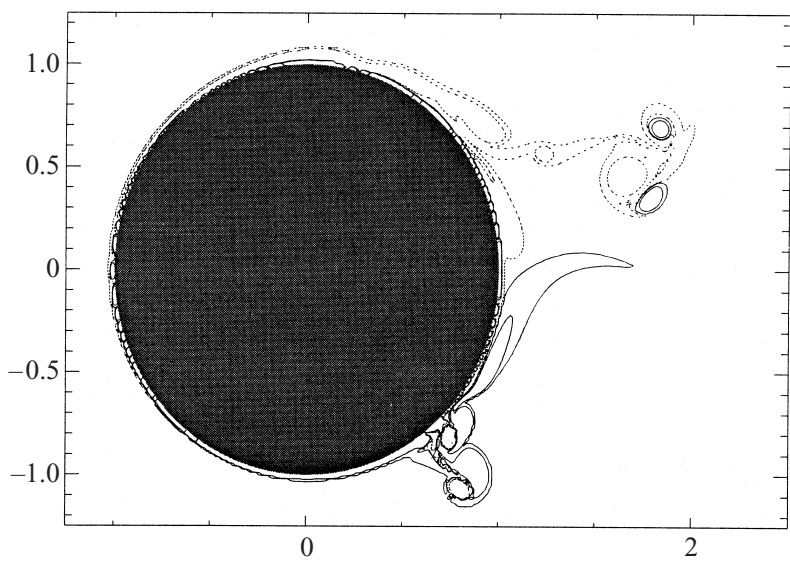

FiguRE 7. Vorticity field for $R e=15000$ flow, $\Omega^{*}=2 \sin \left(\pi t^{*}\right), t^{*}=3.15$, contours at $\omega^{*}= \pm 1, \pm 10, \Delta x^{*} \approx 0.001$ and $\Delta t^{*}=0.0025$.

sharp unsteadiness observed in the drag coefficient appears to be related to the growth period of the multipole vorticity structures near the body. The distribution of pressure around the body at $t^{*}=5$ is shown in figure 5. Pressure is obtained by relating the tangential pressure gradient on the body surface to the normal gradient of the vorticity field with the momentum equation. A relative measure of the pressure can thus be obtained. The precise value in relation to a reference quantity (such as the pressure in the undisturbed free stream) is not determined in this approach. For more details, see Shiels (1998). The pressure distribution clearly indicates a strong recovery, consistent with the reduced level of drag. The shape of the distribution is far from the classical time-averaged shape for a bluff body, namely a flat, low-pressure region after mean separation. The streamlines at $t^{*}=5$ are also plotted in figure 6 .

In order to perform a convergence test on the results above, the same flow was simulated but with the higher resolution $\Delta x^{*} \approx 0.001$ and $\Delta t^{*}=0.0025$. To reach $t^{*}=3.15$, this computation required almost 60 hours of use of 256 processors on the Cray T3D and entailed over 2 million computational particles. The vorticity field which evolved in this computation can be viewed in figure 7 and the resultant drag coefficient in figure 8 . Similarities to the previous simulation $\left(\Delta x^{*} \approx 0.0015\right.$ 


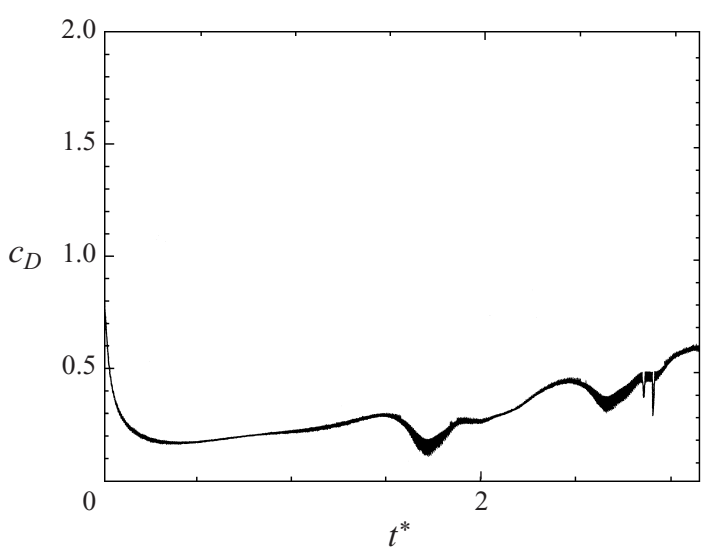

FiguRE 8. Drag for $R e=15000$ simulation, $\Omega^{*}=2 \sin \left(\pi t^{*}\right), \Delta x^{*} \approx 0.001$ and $\Delta t^{*}=0.0025$.

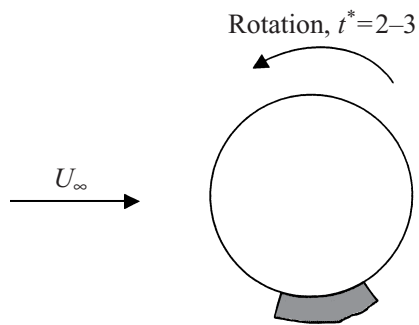

FIGURE 9. Velocity fields in figure 10 are measured over the shaded region.

and $\left.\Delta t^{*}=0.004\right)$ can be seen in both the generally reduced magnitude of the drag coefficient and the generation of the large vortex structures in the vorticity field. However, some smaller scale discrepancies exist between the two vorticity fields, and the drag coefficient in figure 8 does not exhibit the same level of unsteadiness as in figure 4 . The results strongly suggest that the computations have converged to the large-scale dynamics which characterize this flow but have not fully resolved smaller scale features. In an attempt to illuminate the dynamics behind the growth of the multipole vorticity structures, the velocity field inside the boundary layer is examined in figure 10. These fields are discussed below.

\subsection{Analysis of highest-resolution results}

The simulations presented above shed light on the somewhat unusual phenomena which appear to dominate the dynamics of this reduced-drag flow. The observation of multipole vortex structures which erupt from the boundary layer each half-cycle of forcing on alternating sides of the body agrees with the shedding patterns observed in Tokumaru's (1991) work. The existence of both signs of vorticity in each structure and the orientation of the structure induces a local velocity field that draws the structure away from the body in much the same way that a vortex dipole propels itself. The structures thus enter the free stream and convect away quickly rather than agglomerating in the cylinder wake and establishing large-scale separation. Such observations are tempered by the relatively short duration of these computations, but they do agree qualitatively with the experimental results and there is clearly a trend toward periodic behaviour, as exhibited in the comparison of figures $2(b)$ and $2(e)$, and of figures $2(c)$ and $2(f)$. 
(a)

(b)

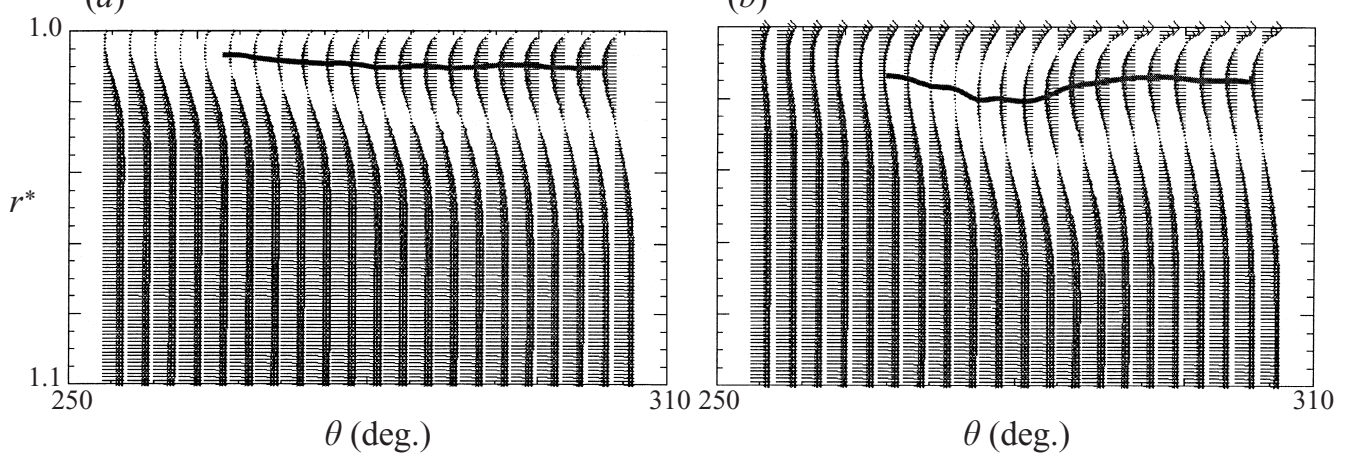

(c)

(d)

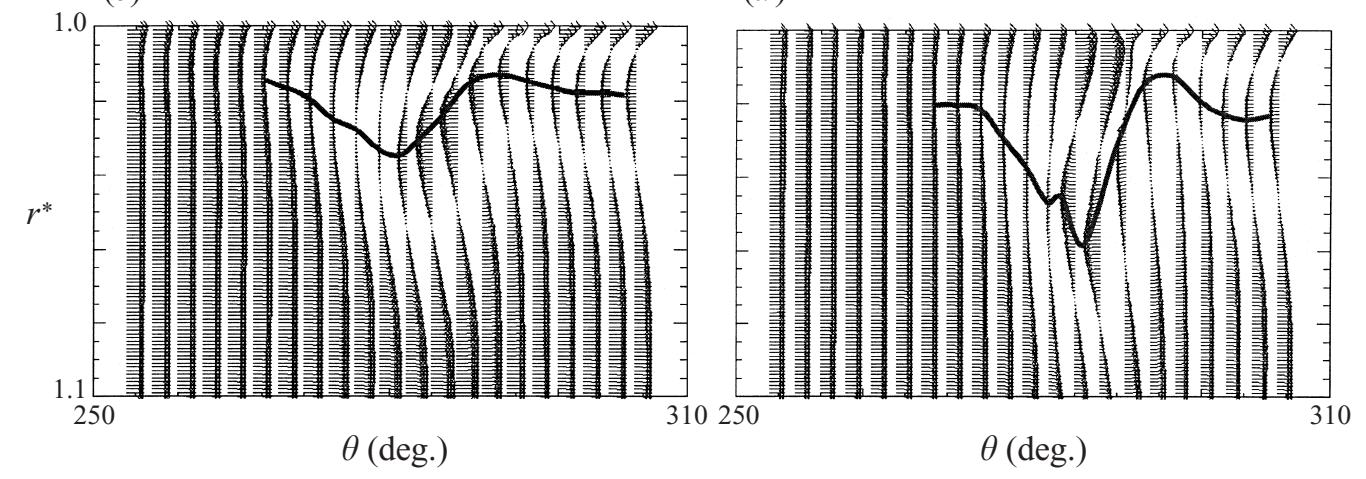

FIGURE 10. Velocity vectors in the boundary layer at $(a) t^{*}=2.05,(b) 2.15,(c) 2.3,(d) 2.6$. The grey line approximates an $\omega^{*}=0$ contour.

It is informative to examine the origins of the multipole vortex structures through boundary layer velocity profiles, measured in the region shown in figure 9. In figure $10(a)$, the boundary layer from the clockwise rotation of $t^{*}=1-2$ can be seen drifting off the surface of the body as the counter-clockwise rotation begins at $t^{*}=2$. The grey line in the figures tracks approximately the line $\omega=0$ and hence the migration of this vortex layer as the counter-clockwise rotation of the $t^{*}=2-3$ cycle frees it from the restrictions of the solid boundary. It appears from the evolution of the grey lines in figure 10 that a compact vortex forms in this location. As seen in figure 10(a), the flow leading up to this bursting event has the ingredients for unsteady boundary layer separation - significant negative velocity gradient in the stream direction along the line $\omega=0$. See, for example, Cowley, Van Dommelen \& Lam (1990). The separation thus triggers rapid growth of the observed multipole vortex structure, which quickly pulls itself outside the boundary layer. All of this is made possible because of the transient effects of the rotational oscillations combined with advection effects, providing the simultaneous existence of opposite-signal vortex layers with variable strength near the wall.

These vorticity dynamics provide a plausible explanation of the observations in Tokumaru's (1991) experiment. The vortex multipole draws a great deal of vorticity far enough from the body to permit it to shed downstream rather than get caught in a recirculation region. By drawing this vorticity out of the boundary layer, sufficient vorticity never remains in the near wake to establish a large-scale recirculation. Presumably the interaction of opposite signs of vorticity in the multipole creation 


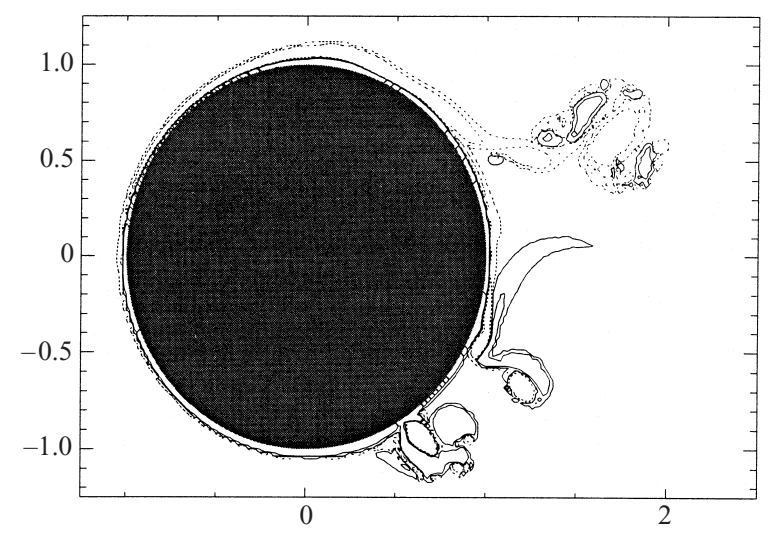

Figure 11. Vorticity field for $R e=15000, \Omega^{*}=2 \sin \left(\pi t^{*}\right), t^{*}=3.15$, contours at $\omega^{*}= \pm 1, \pm 10$. Implementation of the core expansion technique with $\Delta t^{*}=0.005$ (highest resolution).

also leads to a good deal of cancellation. Thus separation is delayed in an overall sense, leading to pressure profiles such as in figure 5, although the creation of the vortex multipoles can be viewed as short-term separation enhancement. Further evidence that separation is being delayed in a time-averaged sense is provided by the tongues of vorticity near the wake centreline in figure $2(f)$, representing the shear layers that would normally have separated from the cylinder much earlier.

Another view of how the flow dynamics observed in the above simulations supports drag reduction is given by considering drag expressed from the vorticity perspective,

$$
\text { Drag }=-\rho \frac{\mathrm{d}}{\mathrm{d} t}\left(\iint_{A} y \omega \mathrm{d} A\right)
$$

where the area $A$ represents the entire flow field. The vortex structures which are shed from the body contain significant components of both signs of vorticity. With comparable vortices of opposing sign at relatively the same location, (13) would thus predict less drag than for the unforced case where structures of one sign of vorticity are more prevalent.

As noted above, the simulations on which this analysis is based are not fully converged. However, the dominant dynamics of the ejection of multipole vorticity structures appear to exist on scales that are properly resolved.

\subsection{Long-time simulations with varied Re and oscillation parameters}

\subsubsection{Application of the core expansion method}

In order to attempt longer-duration simulations of flow at $R e=15000$, a vortex method with the core expansion technique for diffusion is used. The goal of these computations is to simply capture the dominant behaviour observed in the PSE simulations while relaxing the level of resolution to an extent that allows simulation of the flow to a well-developed state. In order to identify appropriate choices for the computational parameters of the core expansion simulations, test cases are run on the $R e=15000, \Omega^{*}=2 \sin \left(\pi t^{*}\right)$ flow. The resultant vorticity fields and drag coefficients are compared to the benchmarks results shown in figures 7 and 8 respectively.

Three sets of parameters were tested to assess the accuracy of the core expansion method for the flow. The highest-resolution case provides confidence that the method will converge towards the PSE results at the relevant scales. This simulation used $\Delta t^{*}=$ 


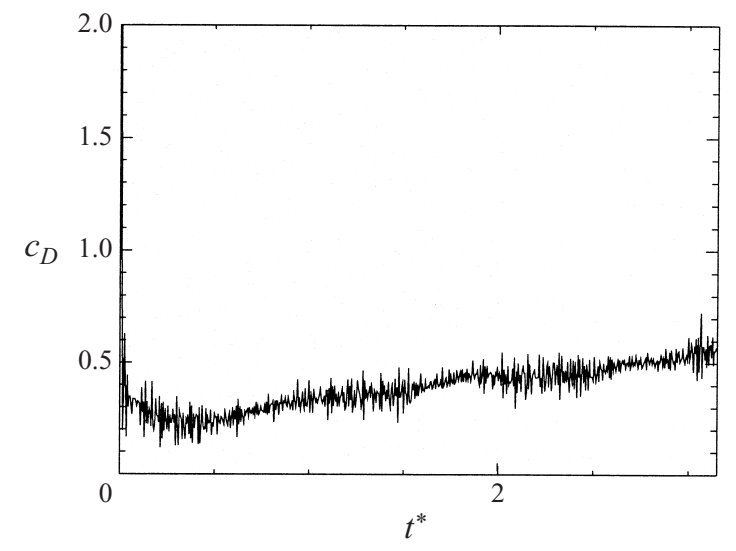

FIGURE 12. Drag for $R e=15000$ flow, $\Omega^{*}=2 \sin \left(\pi t^{*}\right)$. Implementation of the core expansion technique with $\Delta t^{*}=0.005$ (highest resolution).

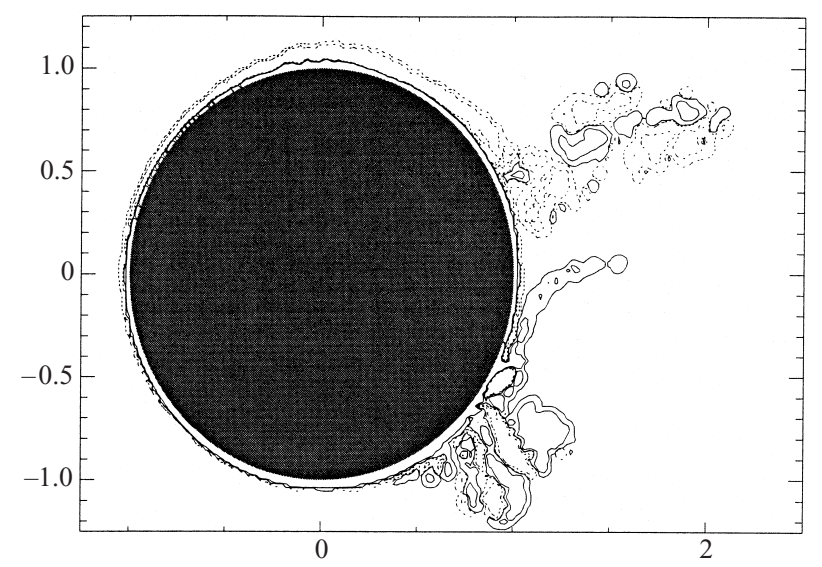

Figure 13. Vorticity field for $R e=15000, \Omega^{*}=2 \sin \left(\pi t^{*}\right), t^{*}=3.15$, contours at $\omega^{*}= \pm 1, \pm 10$. Implementation of the core expansion technique with $\Delta t^{*}=0.01$ (medium resolution).

0.005 , spatial adaptivity from $\mathrm{d} x^{*} \approx 0.0018$ in the boundary layer to $\mathrm{d} x^{*} \approx 0.005 r^{*}$ (where $r$ gives the radial distance from the centre of the cylinder), and required 13 hours on a single processor of a Cray J90 (which is approximately equivalent to 26 hours on a $200 \mathrm{MHz}$ Pentium Pro for the code used). Comparison between vector and parallel computers can be difficult to quantify, but an hour on a single J90 processor could be considered substantially less 'costly' than an hour of use of 256 processors of a Cray T3D. By the end of the simulation, only 170000 computational elements were required compared to the 2 million for the simulation with PSE. The vorticity field in figure 11 exhibits the familiar growth and ejection of multipole vorticity structures, although the structures are a bit more complex than in the PSE simulations. Considering the aggressive spatial resolution adaptation being applied in this case, the field is in good agreement with the PSE simulations, obtaining similar qualitative behaviour. The drag coefficient also exhibits a similar evolution to the PSE results (see figure 12).

A second simulation at medium resolution also captures the generation of multipole vortex structures every half-cycle, but loses quite a bit of accuracy in the small 


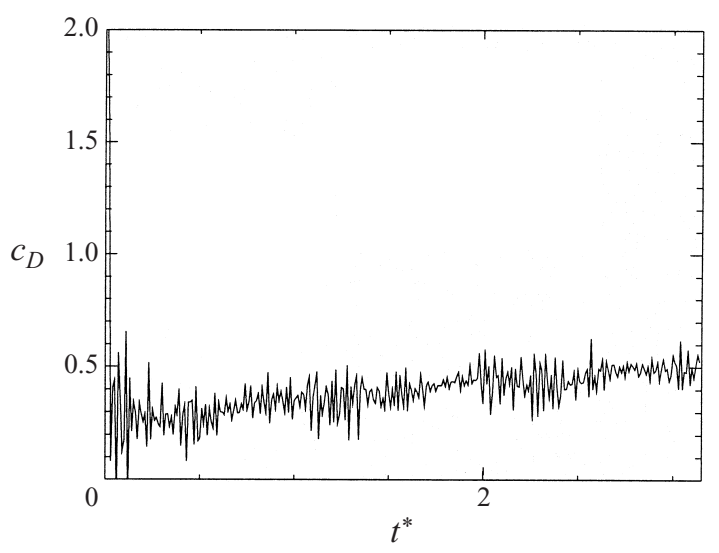

FIGURE 14. Drag for $R e=15000$ flow, $\Omega^{*}=2 \sin \left(\pi t^{*}\right)$. Implementation of the core expansion technique with $\Delta t^{*}=0.01$ (medium resolution).

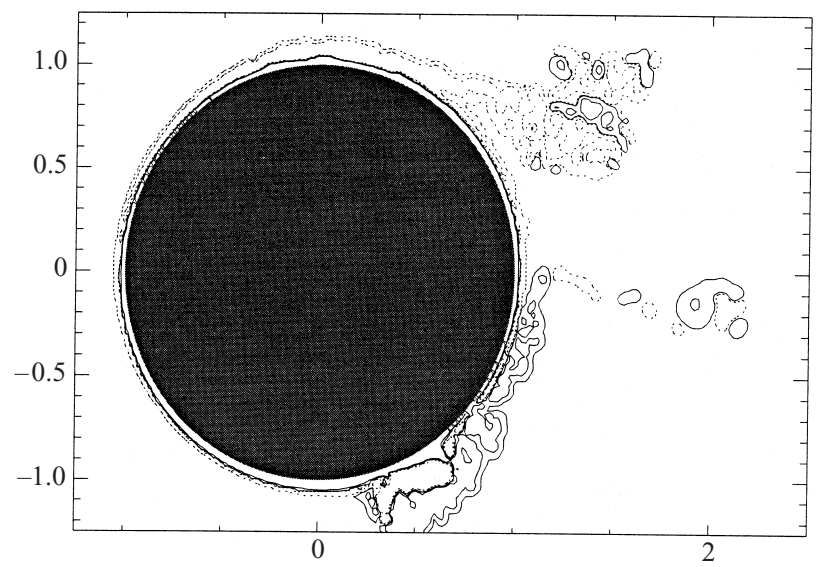

FIGURE 15 . Vorticity field for $R e=15000, \Omega^{*}=2 \sin \left(\pi t^{*}\right), t^{*}=3.15$, contours at $\omega^{*}= \pm 1, \pm 10$ Implementation of the core expansion technique with $\Delta t^{*}=0.02$ (lowest resolution).

scale. This computation used $\Delta t^{*}=0.01$, spatial adaptivity from $\mathrm{d} x^{*} \approx 0.0025$ in the boundary layer to $\mathrm{d} x^{*} \approx 0.015 r^{*}$, and required 2 hours on a single processor of a Cray J90 and 20000 computational elements. Figure 14 indicates that the reduced-drag aspect of the flow is still captured by the medium resolution of the dynamics given by the field in figure 13 .

The lowest-resolution simulation is implemented with the parameters $\Delta t^{*}=0.02$, spatial adaptivity from $\mathrm{d} x^{*} \approx 0.0036$ in the boundary layer to $\mathrm{d} x^{*} \approx 0.02 r^{*}$, and required under one hour on a single processor of a Cray J90 and 12000 computational elements. Here a critical point in the required resolution is apparently exceeded and the basic dynamics of the flow change. The rotation still forces the shedding of a vortex structure each half-cycle, but in figure 15 it can be seen that the structure is dominated by a single sign of vorticity and is not ejected as far from the body. Thus it is possible that it has not actually been shed downstream. The growth of a multipole vorticity structure on the lower half of the cylinder is also clearly modified by the lower resolution as again there is less interaction between the opposing signs of vorticity. Figure 16 indicates that the drag is increased by the modified dynamics 


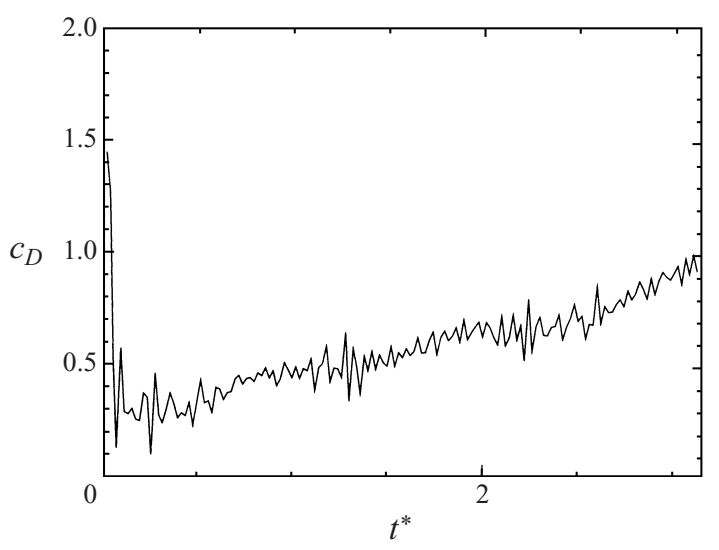

FIGURE 16. Drag for $R e=15000$ flow, $\Omega^{*}=2 \sin \left(\pi t^{*}\right)$. Implementation of the core expansion technique with $\Delta t^{*}=0.02$ (lowest resolution).

resulting from the lower resolution. This indicates that the drag reduction suggested in the PSE simulations is linked to the distinctive vortex shedding.

Subsequent core expansion simulations in this paper use the parameters for the $\Delta t^{*}=0.01$ simulation as it seems to capture the crucial dynamics while gaining much computational efficiency. Resolution in the far wake is limited to a maximum coarseness of $\mathrm{d} x^{*}=0.25$.

\subsection{2. $R e=15000$ flows: effects of variable forcing}

The core expansion method selected above is used to simulate $R e=15000$ flow with forcing of $\Omega^{*}=2 \sin \left(\pi t^{*}\right)$ to $t^{*}=50$, at which point the system has clearly settled into periodic, long-time behaviour. The flow is distinguished by the ejection of a multipole vorticity structure every half-cycle and exhibits a greatly reduced drag. The evolution of the vorticity field over the half cycle $t^{*}=50-51$ exhibits the same general behaviour as the first shed structures seen in the starting-flow simulations above. As presented in figures 17(a) to 17(e), a structure evolves on the lower half of the cylinder, where the rotational motion parallels the free stream. The structure from the previous half-cycle on the upper half of the cylinder is ejected into a wake which exhibits no large-scale separation. Note again the parallels between the flow at $t^{*}=50$ in figure $17(a)$ and the flow at $t^{*}=51$ in figure $17(e)$.

The drag is significantly reduced from the experimentally determined values for a fixed cylinder as seen in figure 18. Averaged over five forcing cycles from $t^{*}=40$ to 50 , the average drag coefficient is $c_{D}=0.43$. Although this is double the Tokumaru (1991) measurement, it represents nearly a 70\% reduction in drag from fixed-cylinder experimental measurements. Experiments include three-dimensional effects, but the two-dimensional computational results capture the same qualitative behaviour as Tokumaru's (1991) experiments and thus seen to reflect the behaviour he observed.

Simulations with different forcing parameters were run to aid in assessing the mechanism driving the observed dynamics. First the amplitude of the oscillation was reduced to $\Omega^{*}=0.5 \sin \left(\pi t^{*}\right)$. In this case the average drag coefficient rose to $c_{D}=1.42$. The vorticity field in figure $19(a)$ reveals that vortex structures shed every step consist primarily of a single sign of vorticity. The wake exhibits a larger-scale vortex structure of positive vorticity immediately behind the body and one of negative vorticity a few diameters downstream in the wake. Thus the altered shedding seems 

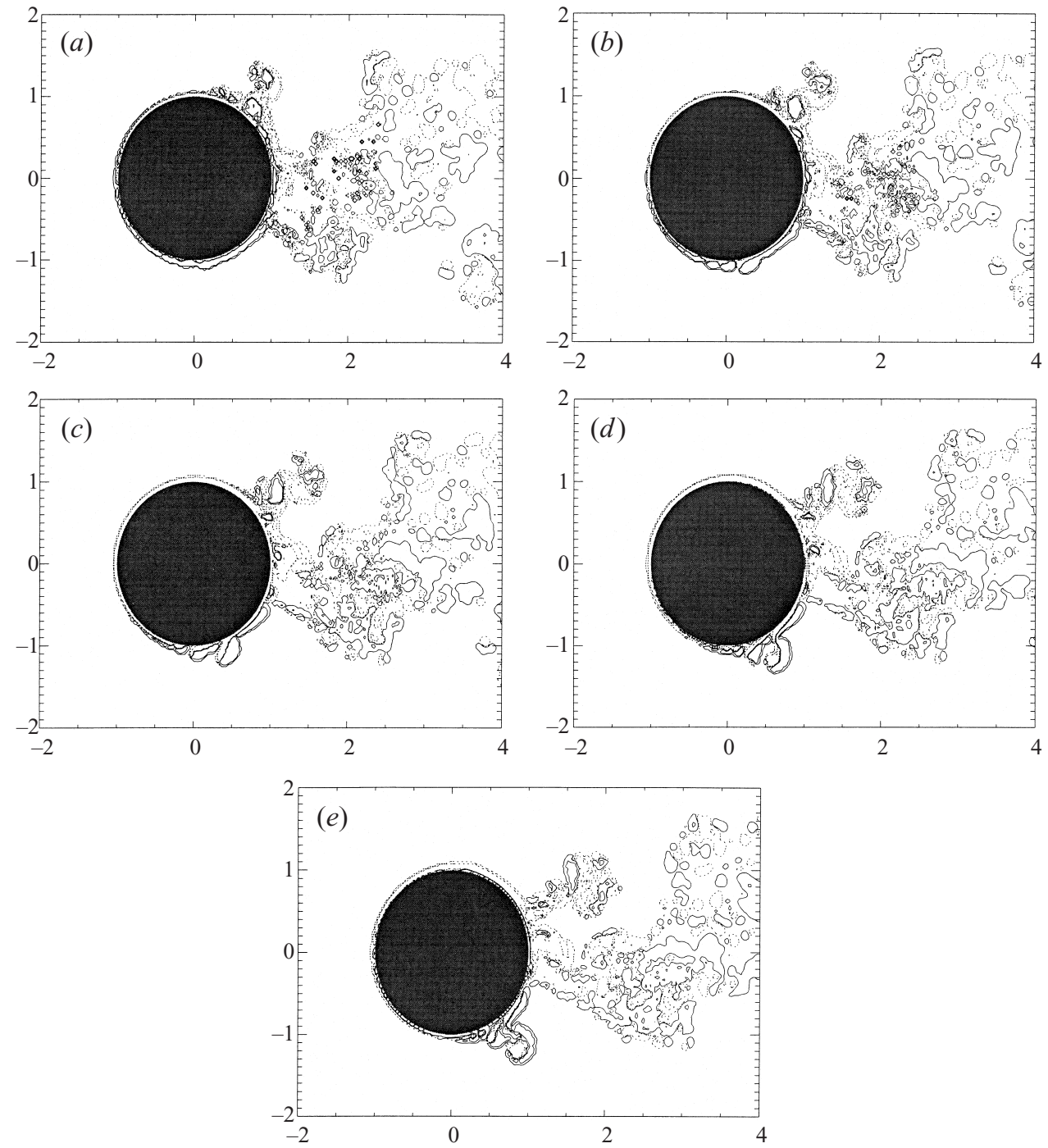

FIGURE 17. Vorticity field for $R e=15000$ flow, $\Omega^{*}=2 \sin \left(\pi t^{*}\right), t^{*}=50.25$, contours at $\omega^{*}= \pm 1$, $\pm 10 .(a) t^{*}=50,(b) 50.25,(c) 50.5,(d) 50.75$, (e) 51.0.

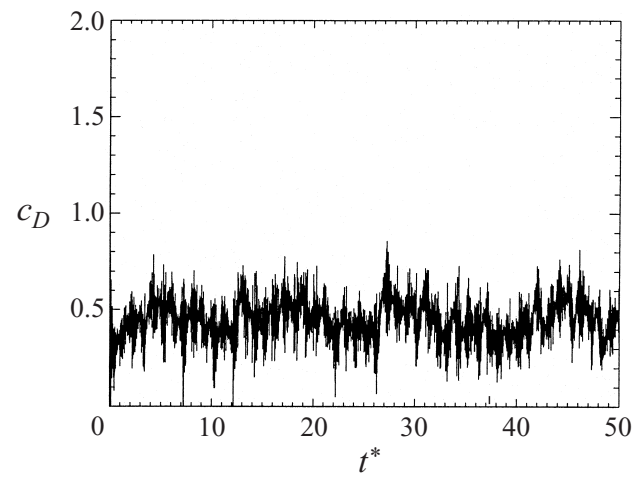

FiguRE 18. Drag for $R e=15000$ flow, $\Omega^{*}=2 \sin \left(\pi t^{*}\right)$. 

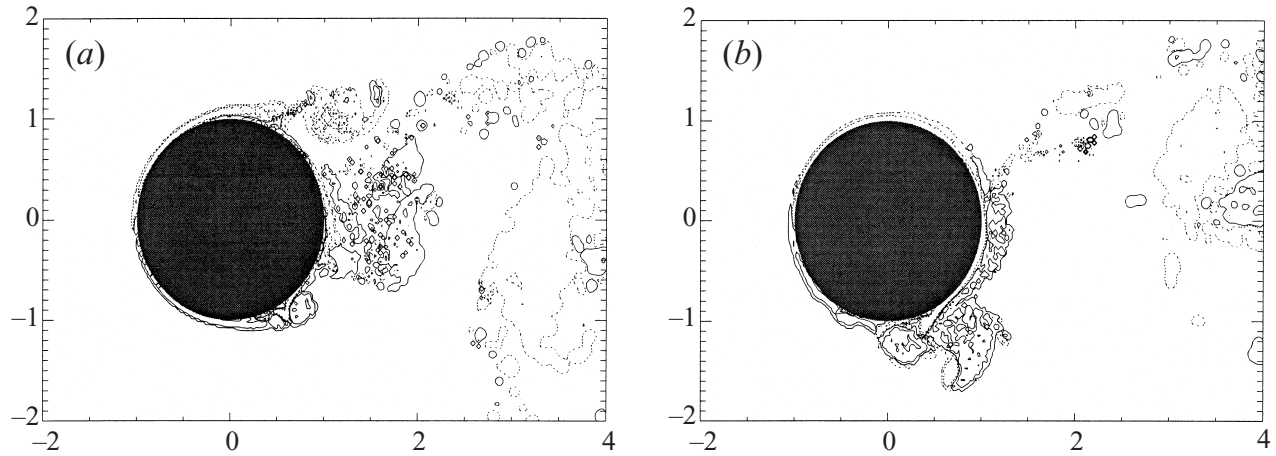

FIgURE 19. Vorticity field for $R e=15000$ flow, $\Omega^{*}=2 \sin \left(0.5 \pi t^{*}\right), t^{*}=48$, contours at $\omega^{*}= \pm 1, \pm 10$. (a) $t^{*}=49,(b) 48$.

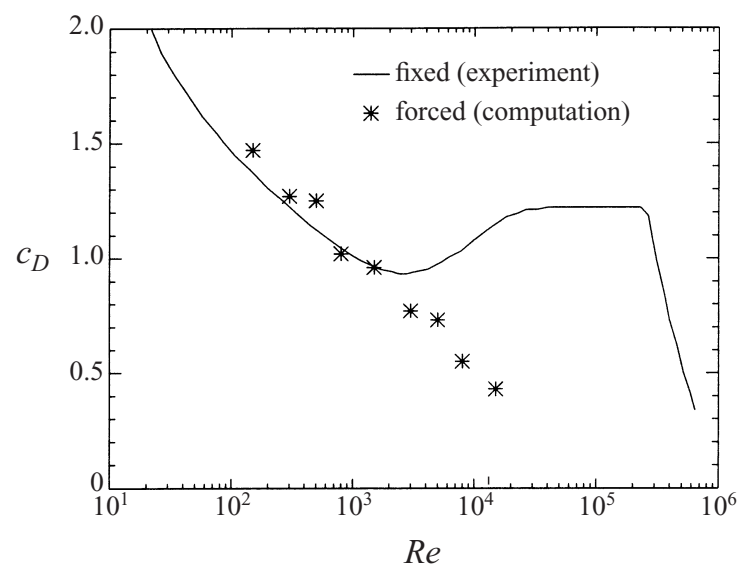

FIGURE 20. Drag measured in two-dimensional simulations with $\Omega^{*}=2 \sin \left(\pi t^{*}\right)$ (symbols) compared to experimental measurements of drag over a fixed cylinder $\left(\Omega^{*}=0\right)$ (line) (Roshko 1961).

to result in more classical wake development. A second case lowers the frequency of the forcing to $\Omega^{*}=2 \sin \left(0.5 \pi t^{*}\right)$, resulting in a drag averaged over a single forcing cycle $\left(t^{*}=40-48\right)$ of $c_{D}=1.14$. The plot of the vorticity field for this case in figure 19 (b) reveals that the vortex multipole creation observed in the other forcing cases no longer exists for this oscillation. The large structure seen on the lower half of the cylinder is presumably the result of the clockwise rotation of the body from $t^{*}=44$ to 48 , during which the body on the lower half moves in opposition to the free stream, enhancing separation as expected in classical thinking.

\subsubsection{Reynolds number effects for $\Omega_{1}^{*}=2, S_{f}=1$}

The influence of Reynolds number on the observed drag reduction is assessed with a series of simulations using the forcing $\Omega^{*}=2 \sin \left(\pi t^{*}\right)$ and identical simulation parameters to the above core expansion tests. Reynolds numbers in the range 150 15000 are considered, with $\Delta t^{*}=0.01$ and spatial adaptivity from $\Delta x^{*} \approx 0.3 / \sqrt{R e}$ in the boundary layer to $\Delta x^{*} \approx(0.9 / \sqrt{R e}) r^{*}$ with the coarsest resolution limited to $\Delta x^{*}=0.25$. The results reveal that both the drag reduction and multipole vortex 

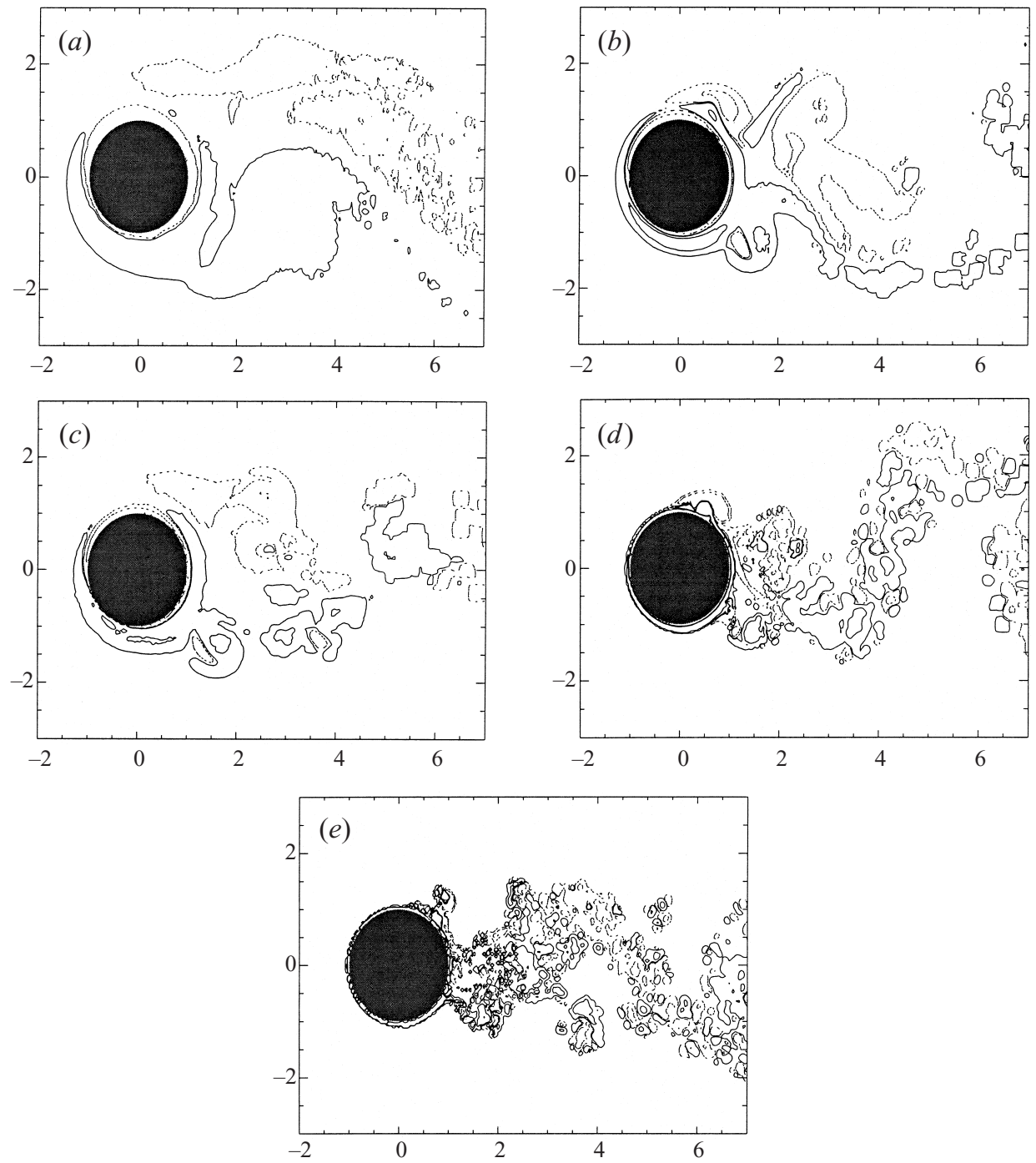

FIgURE 21. Vorticity fields at $t^{*}=50, \Omega^{*}=2 \sin \left(\pi t^{*}\right)$ for $(a) \operatorname{Re}=150,(b) 500,(c) 1500,(d) 5000$, (e) 15000 . Contours at $\omega^{*}= \pm 0.5, \pm 5$ with solid for positive, dashed for negative.

structures only exist at higher Reynolds numbers, and become more apparent as Reynolds number increases.

In order to reach the quasi-periodic state of these flows, simulations were run to $t^{*}=50$ (25 diameters of downstream motion) and averages were taken over five forcing cycles from $t^{*}=40$ to 50 . The average drag coefficient in each case is given in figure 20. At lower Reynolds numbers the forcing is ineffective and the decrease in drag simply parallels the known drop for the fixed cylinder (although for two-dimensional flow, fixed cylinder drag actually increases over the range $R e=200$ 1000; cf Henderson 1995). But at the higher Reynolds numbers, clear drag reduction develops between the forced and fixed cases, increasing to the previously noted significant reduction for $R e=15000$.

Views of the vorticity field at $t^{*}=50$, time-averaged streamlines, and time-averaged 


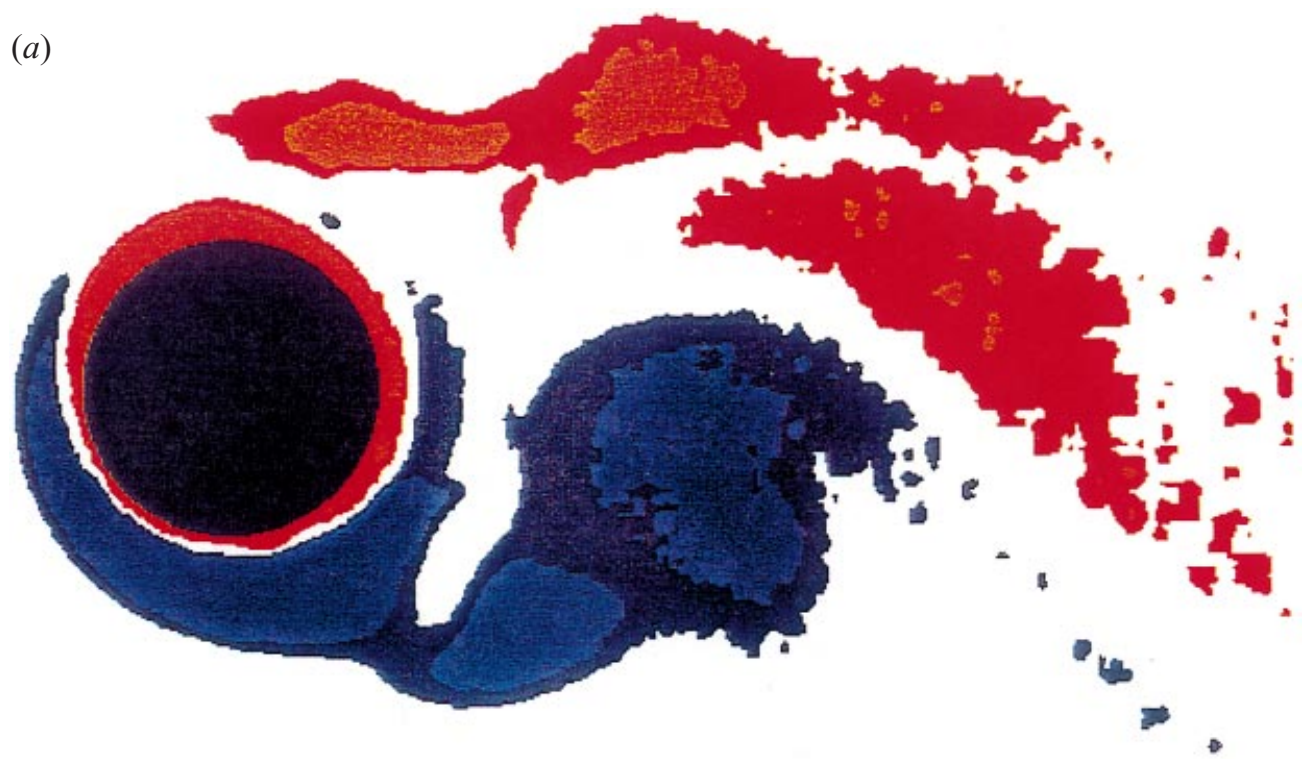

(b)

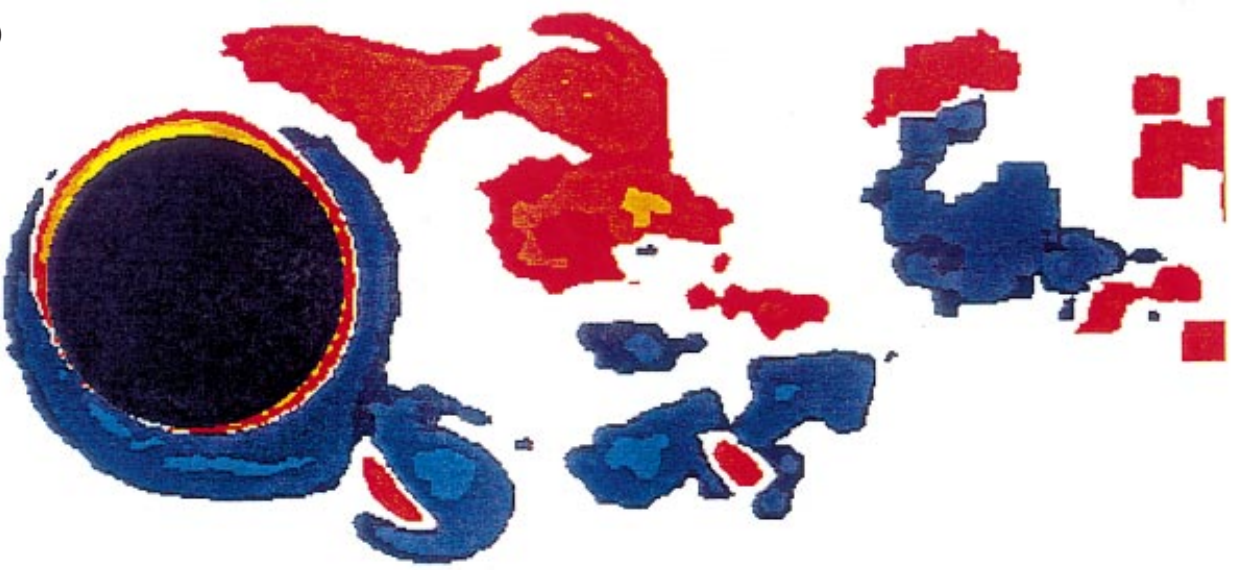

(c)

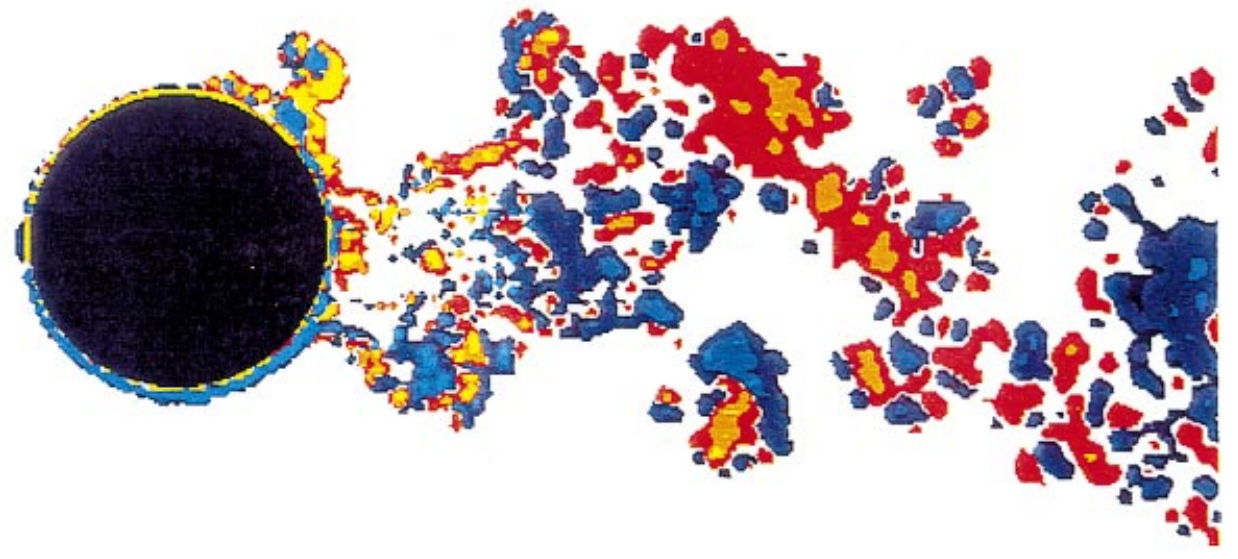

FiguRE 22. Colour plots for (a) $R e=150$, (b) $R e=1500$, and (c) $R e=15000$. 

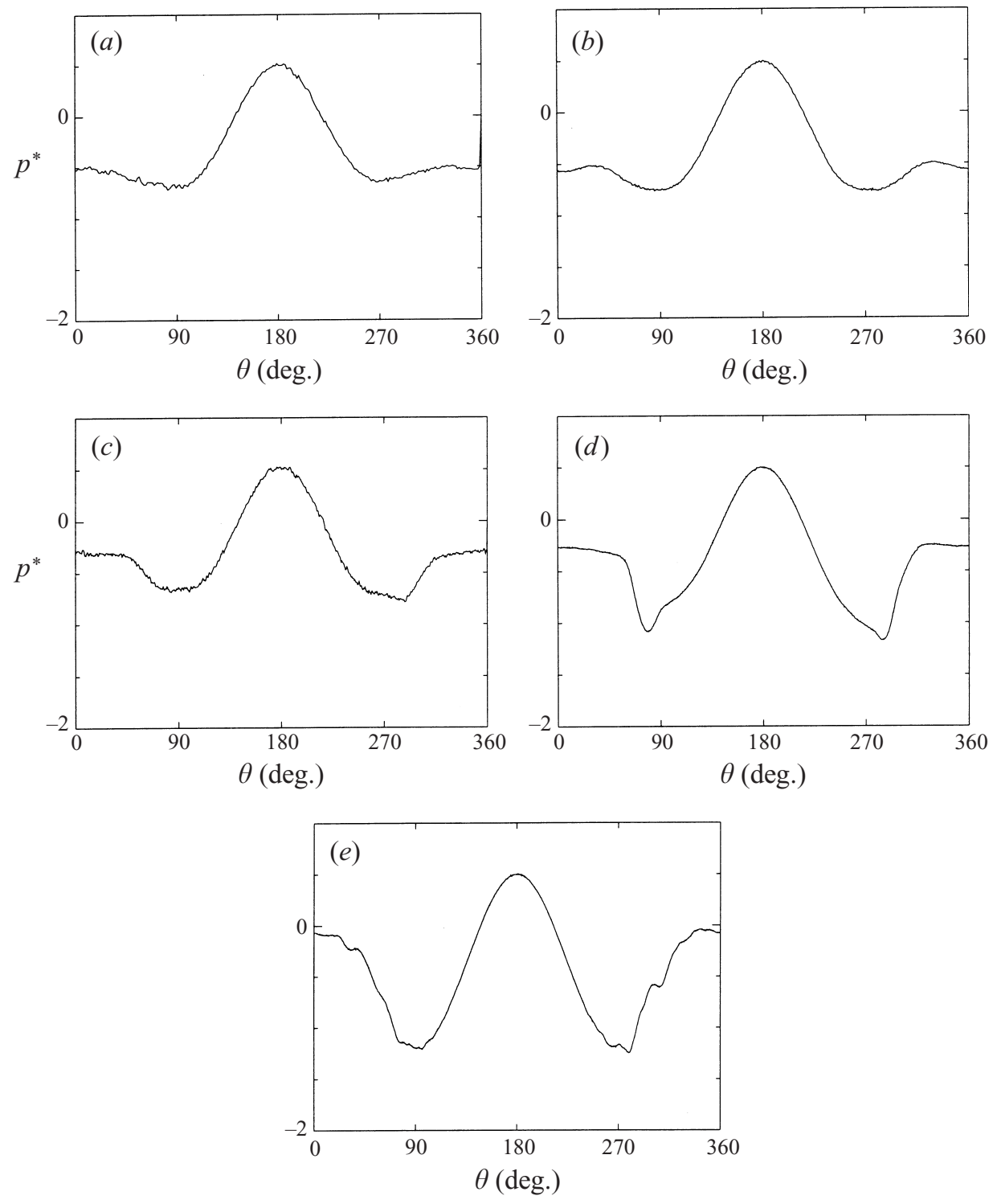

FIGURE 23. Time-averaged pressure distributions with reference value $p^{*}\left(\theta=180^{\circ}\right)=0.5$.

(a) $R e=150,(b)$ 500, (c) 1500, (d) 5000, (e) 15000.

pressure give a good perspective on the foundation of the drag behaviour. At $t^{*}=50$, a half-cycle of forcing with clockwise rotation has been completed and thus a vorticity structure such as that observed in the $R e=15000$ case has formed on the upper half of the cylinder. As the Reynolds number drops, the added diffusivity impedes the roll-up of this structure as seen in figures 21 and 22, and prevents it from attaining the multipole nature evident at $R e=15000$. Between $R e=1500$ and 5000, there is a clear transition in the nature of the development of this structure, just as the drag reduction begins to become significant in this range.

At the lower Reynolds numbers, the vortex structures formed every half forcing cycle could be characterized as the shedding of a slight dipole dominated by a single 

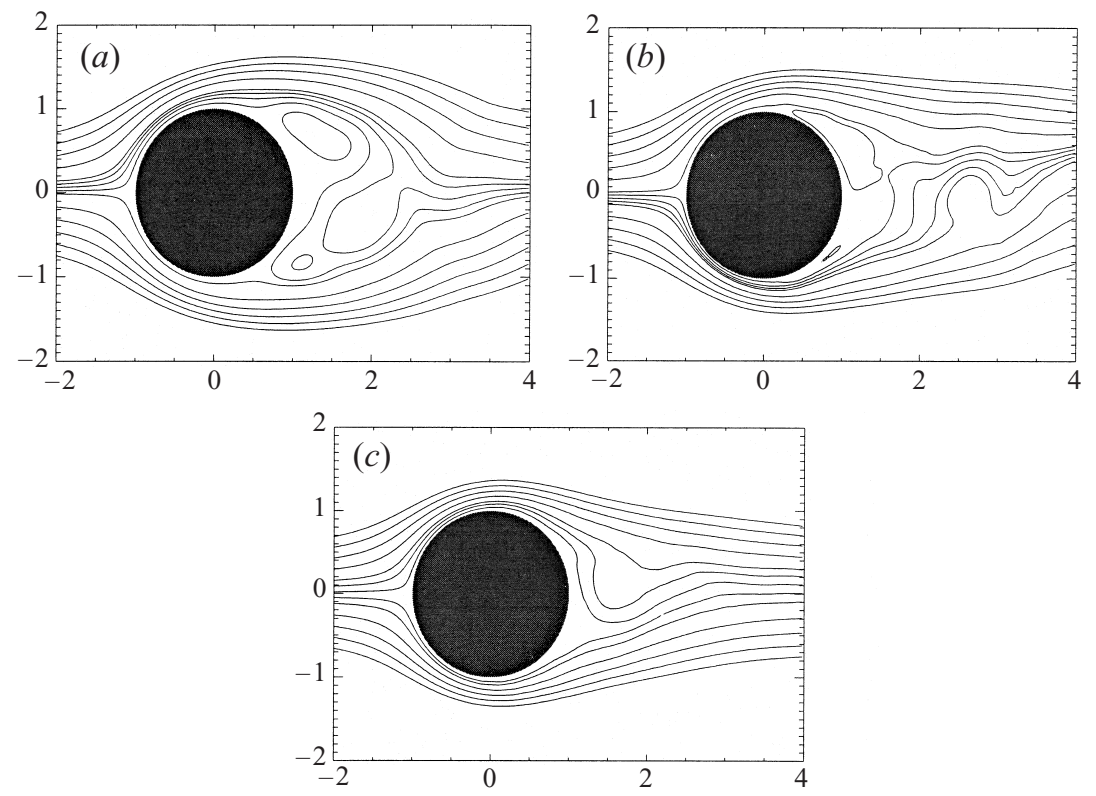

FiguRE 24. Time-averaged streamlines, contours at $\psi^{*}=0, \pm 0.1, \pm 0.2, \pm 0.3, \pm 0.4, \pm 0.5$. (a) $R e=500,(b)$ 5000, (c) 15000 .

sign of vorticity. For $R e=5000$, the structures become more complex. Note how the structure formed on the lower half of the cylinder from $t^{*}=48$ to 49 is drawn farther from the body as Reynolds number increases. And note how at low Reynolds numbers the structures dominated by a single sign of vorticity tend to clump into large-scale vortices in the wake, typical of natural vortex shedding. On the other hand, the mixed-sign multipole structures at the higher Reynolds numbers lead to a more heterogeneous near-wake dominated by smaller vortex structures.

The vortex dynamics can be seen as linked to a general trend of a time-averaged delay in separation as Reynolds number increases, thus leading to drag reduction. The separation delay occurs only in a time-averaged sense as the rotation triggers frequent, small-scale, unsteady separation events. The time-averaged pressure distributions in figure 23 clearly show a pattern of increasing time-averaged delay of separation as Reynolds number is increased. Time-averaged streamlines in figure 24 verify that the flow field exhibits this time-averaged form of separation delay at the higher Reynolds numbers.

It is again instructive to observed the boundary layer developments in the region of multipole vortex creation in figures 25 to 27. At both $R e=15000$ and $R e=5000$, similar dynamics to those observed in the short-time $R e=15000$ simulation above are present. In both cases, the boundary layer from the previous rotation half-cycle becomes a strong, relatively unconstrained shear layer and a compact vortex rolls up in this region, leading to the multipole development. At $R e=1500$, however, this region of large shear and reversed flow does not exist, presumably due to the greater influence of diffusion at this Reynolds number. Similarly, the suppression of multipole growth for lower forcing amplitude and frequency at $R e=15000$ observed above is presumably linked to the absence of a thin, opposite-signed vortex layer residing above the wall layer as observed in figures 25 and 26.

Observing the rotational forcing over a range of Reynolds numbers helps to 
(a)

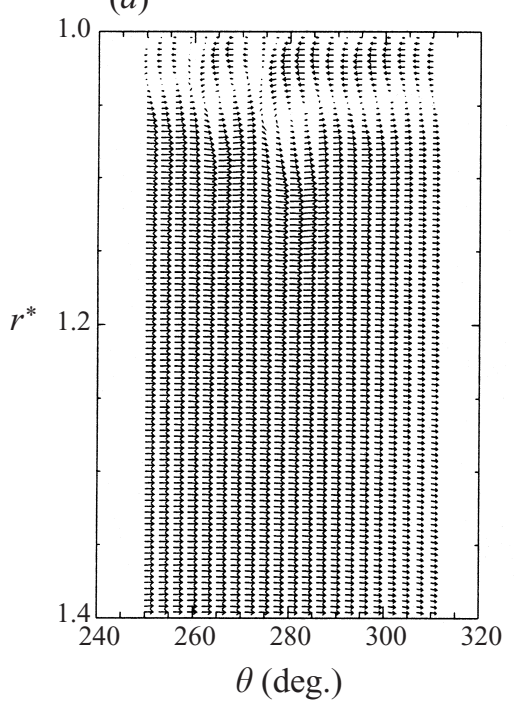

(b)

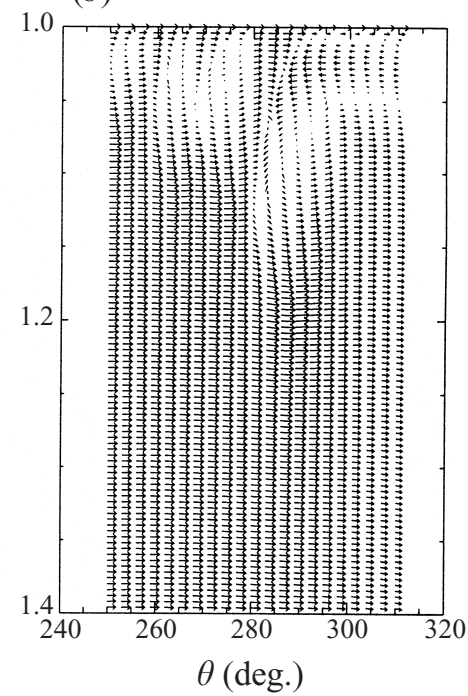

FigURE 25. Velocity profiles in the boundary layer for $R e=15000,(a) t^{*}=50.05$ and $(b) 50.3$.

(a)

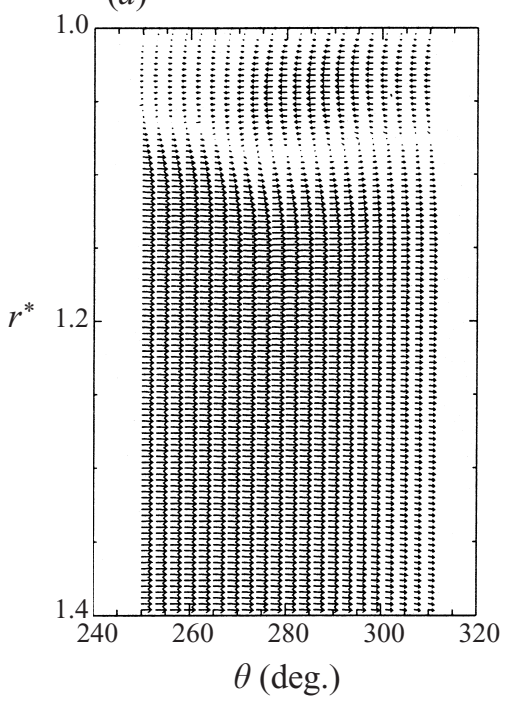

(b)

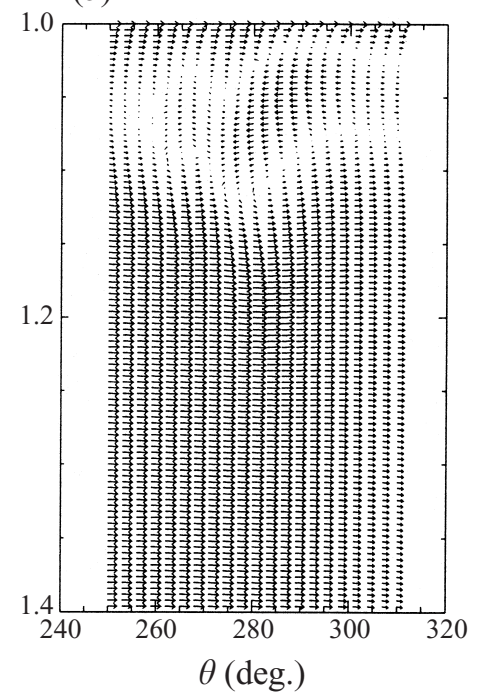

FigURE 26. Velocity profiles in the boundary layer for $R e=5000,(a) t^{*}=50.05$ and (b) 50.3 .

clarify the physics behind measured drag reductions. The vortex multipole found to grow in the boundary layer for the $R e=15000$ simulations encourages an overall delay in separation, presumably by a combination of destruction of vorticity through cancellation and convection of vorticity away from stagnation regions. As Reynolds number is decreased and diffusivity increases, the multipole growth is lessened and eventually suppressed, thus reducing its effects and leading to less drag reduction. An understanding of the dynamics behind the drag reduction also clearly indicates that a laminar-to-turbulent boundary layer transition is not responsible for the lowered drag. 
(a)

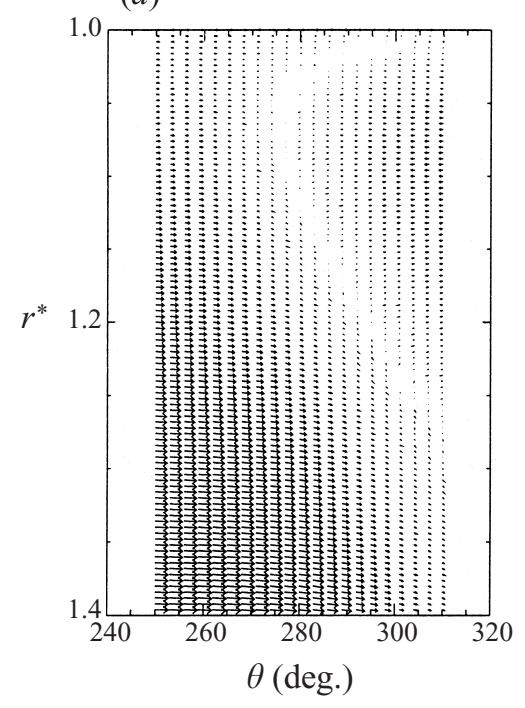

(b)

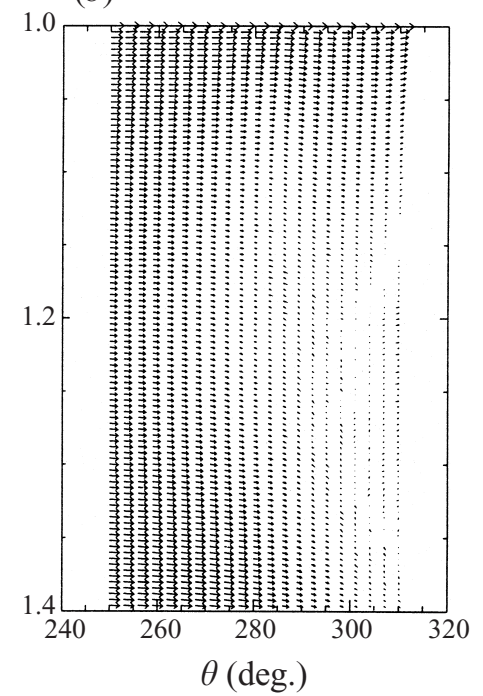

FIGURE 27. Velocity profiles in the boundary layer for $R e=1500,(a) t^{*}=50.05$ and $(b) 50.3$.

\subsubsection{Efficiency of control at $\Omega_{1}^{*}=2, S_{f}=1$}

A final issue concerns the practical efficiency of the rotational control studied above. Although drag reduction can be obtained, there is a cost associated with rotating the cylinder against skin friction. A comparison is made between the power saved (which otherwise would be expended to move the body upstream) by the drag reduction and the power required to rotate the cylinder against skin friction. There is further cost in overcoming the rotational inertia of the body, but this is dependent on material properties and practical implementation and not factored into this analysis. Define the power savings ratio, PSR, as the ratio between the power saved by drag reduction and power expended by the rotation. The power saved by the drag reduction is given by

$$
\text { Power saved }=\left(\frac{1}{2} \rho U_{\infty}^{2} D \Delta c_{D}\right) U_{\infty}=\frac{1}{2} \rho U_{\infty}^{3} D \Delta c_{D} .
$$

The power exerted to rotate against skin friction is given by

$$
\text { Power exerted }=\left(\rho U_{\infty}^{2} R^{2} c_{M}\right) \dot{\theta}
$$

where the moment coefficient

$$
c_{M}=-\frac{1}{R e}\left(\int_{0}^{2 \pi} \omega^{*} \mathrm{~d} \theta-4 \pi \dot{\theta}^{*}\right) \mathrm{d} \theta .
$$

Thus the power savings ratio is given by

$$
P S R=\frac{\Delta c_{D}}{\left|\dot{\theta}^{*} c_{M}\right|}
$$

where the denominator is time averaged as for the drag coefficient. Figure 28 shows that the efficiency of the process begins to greatly increase in as the Reynolds number rises to 15000 .

In table 1 a summary of the results from the long-time simulations with the core expansion method is provided. 


\begin{tabular}{rrcc}
\hline Re & \multicolumn{1}{c}{$\Omega^{*}$} & $c_{D}$ & $\dot{\theta}^{*} c_{M}$ \\
150 & $2 \sin \left(\pi t^{*}\right)$ & 1.47 & 0.84 \\
300 & $2 \sin \left(\pi t^{*}\right)$ & 1.27 & 0.56 \\
500 & $2 \sin \left(\pi t^{*}\right)$ & 1.25 & 0.42 \\
800 & $2 \sin \left(\pi t^{*}\right)$ & 1.02 & 0.32 \\
1500 & $2 \sin \left(\pi t^{*}\right)$ & 0.96 & 0.27 \\
3000 & $2 \sin \left(\pi t^{*}\right)$ & 0.77 & 0.16 \\
5000 & $2 \sin \left(\pi t^{*}\right)$ & 0.73 & 0.13 \\
8000 & $2 \sin \left(\pi t^{*}\right)$ & 0.55 & 0.10 \\
15000 & $2 \sin \left(\pi t^{*}\right)$ & 0.43 & 0.07 \\
15000 & $0.5 \sin \left(\pi t^{*}\right)$ & 1.42 & 0.02 \\
15000 & $2 \sin \left(0.2 .5 \pi t^{*}\right)$ & 1.14 & 0.03
\end{tabular}

TABLE 1. Results for simulations with rotational forcing run to $t^{*}=50$ with the core expansion method.

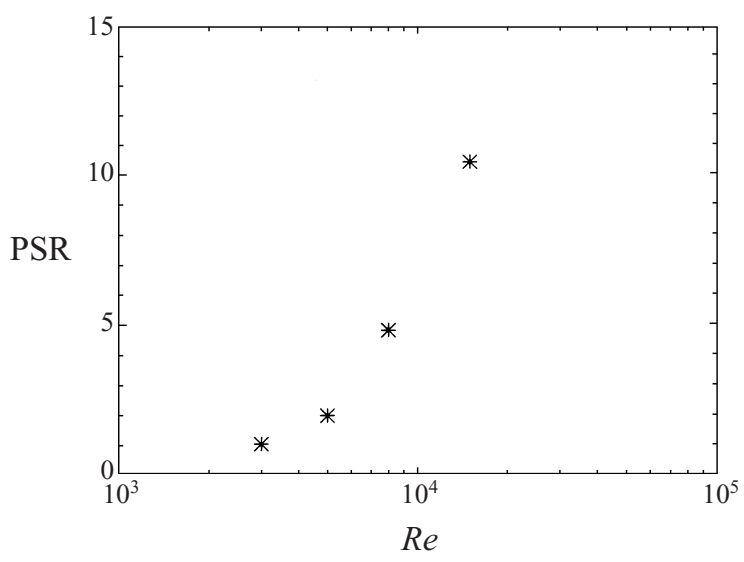

FIGURE 28. The power saving ratio (PSR) for cases in which drag was substantially reduced.

\section{Conclusions}

The computational simulations presented verify Tokumaru \& Dimotakis' (1991) observation of drag reduction. Analysis of the data shows that boundary layer bursting leading to the formation of a multipole vorticity structure, triggered by appropriate rotational oscillation, leads to a time-averaged separation delay and thus drag reduction. This control mechanism is only found to be effective at higher Reynolds numbers ( $R e \gtrsim 3000$ ), apparently because viscosity damps the trigger to the multipole growth. Similarly, it is found that the amplitude and frequency of the forcing must be sufficiently large to generate the multipoles, which coincides with Tokumaru's (1991) observations. The cause of these two limitations on the control effectiveness seems to stem from the link between the multipole structure and its precursor, a boundary layer composed of two opposite-signed vortex layers of variable strength. Convergence tests are used to verify that a sufficient level of accuracy is present in the computations. Although the smallest scales are beyond the level of resolution achieved in this study, it is shown that the dynamics accurately capture qualitative behaviour in the flow and drag measurement is converged. Finally, the efficiency of 
rotational control for drag reduction is assessed, suggesting that this procedure is most efficient at higher Reynolds numbers.

We would like to thank Dr Alan Stagg for his assistance and Dr Stephen Cowley for informative discussions. This work has been supported by ONR Grant \#N00014-941-0793. Computing time was partially provided by the JPL Supercomputing Project (funded from the NASA Offices of Mission to Planet Earth, Aeronautics, and Space Sciences). Scholarship support was provided under DoD Grant \#DAAH04-93-G0281.

\section{REFERENCES}

BAEK, S. \& Sung, H. 1998 Numerical simulation of the flow behind a rotary oscillating circular cylinder. Phys. Fluids 10, 869.

CHou, M. 1997 Synchronization of vortex shedding from a cylinder under rotary oscillation. Computers Fluids 26, 755.

Cowley, S., Van Dommelen, L. L. \& Lam, S. T. 1990 On the use of lagrangian variables in descriptions of unsteady boundary-layer separation. Phil. Trans. R. Soc. Lond. 333, 343.

Degond, P. \& Mas Gallic, S. 1989 The weighted particle method for convection-diffusion equations. Math Comput 53, 485.

EL-Refaee, M. M. 1995 Vortex lock-on for a rotationally oscillating circular cylinder-a BEM numerical study. Engng Anal. Boundary Elements 15, 235.

Filler, J. R., Marston, P. L. \& Mih, W. C. 1991 Response of the shear layers separating from a circular cylinder to small-amplitude rotational oscillations. J. Fluid Mech. 231, 481.

GreENGARD, C. 1985 The core spreading vortex method approximates the wrong equation. J. Comput. Phys. 61, 345.

Griffin, O. M. \& Hall, M. S. 1991 Review-vortex shedding lock-on and flow control in bluff body wakes. Trans. ASME: J. Fluids Engng 113, 526.

Henderson, R. 1995 Details of the drag curve near the onset of vortex shedding. Phys. Fluids 7, 2102.

Koumoutsakos, P. \& Leonard, A. 1995 High-resolution simulations of the flow around an impulsively started cylinder using vortex methods. J. Fluid Mech. 296, 1.

Koumoutsakos, P., Leonard, A. \& Pepin, F. 1994 Boundary conditions for viscous vortex methods. J. Comput. Phys. 113, 52

Koumoutsakos, P. \& Shiels, D. 1996 Simulations of the viscous flow normal to an impulsively started and uniformly accelerated flat plate. J. Fluid Mech. 328, 177.

Leonard, A. 1980 Vortex methods for flow simulation. J. Comput. Phys. 37, 289.

OKajima, A., TaKata, H. \& Asanuma, T. 1975 Viscous flow around a rotationally oscillating circular cylinder. Inst. Space Aeronaut. Sci. Univ. Tokyo Rep. 532.

Ou, Y. 1991 Control of oscillatory forces on a circular cylinder by rotation. NASA Contractor Rep. 187619; ICASE Rep. 91-67.

Roshko, A. 1961 Experiments on the flow past a cylinder at very high Reynolds number. J. Fluid Mech. 10, 345.

Rossi, L. 1996 Resurrecting core spreading vortex methods: a new scheme that is both deterministic and convergent. SIAM J. Sci. Comput. 17, 370.

Rossi, L. 1997 Merging computational elements in vortex simulations. SIAM J. Sci. Comput. 18, 1014.

SHIELS, D. 1998 Simulation of controlled bluff body flow with a viscous vortex method. PhD thesis, Caltech.

Shiels, D., Leonard, A. \& StagG, A. 1995 Computational investigation of drag reduction on a rotationally oscillating circular cylinder. 2nd Intl Work Shop on Vortex Flows and Related Numerical Methods., Montreal, Canada (electronically published in: ESAIM Proceedings, Vol. 1, http://www.emath.fr/Maths/Proc/Vol.1/shiels.htm).

Spalart, P. 1982 Numerical simulation of separated flows. PhD thesis, Stanford University. 
TANEDA, S. 1978 Visual observations of the flow past a circular cylinder performing a rotary oscillation. J. Phys. Soc. Japan 45, 1038.

ToKumaru, P. T. 1991 Active control of the flow past a cylinder executing rotary motions. PhD thesis, Caltech.

Tokumaru, P. T. \& Dimotakis, P. E. 1991 Rotary oscillation control of a cylinder wake. J. Fluid Mech. 224, 77.

Wu, J. M., Mo, J. D. \& VAKILI, A. D. 1989 On the wake of a cylinder with rotational oscillations. AIAA Paper 89-1024.

ZAKHARENKov, M. N. 1996 Unsteady detached separation from a circular cylinder performing rotational oscillations in a uniform viscous incompressible flow. Intl J. Numer. Meth. Fluids $25,125$. 